\title{
Supersymmetric solutions of 7D maximal gauged supergravity
}

\author{
Parinya Karndumri॰* and Patharadanai Nuchino $\odot^{\dagger}$ \\ String Theory and Supergravity Group, Department of Physics, Faculty of Science, \\ Chulalongkorn University, 254 Phayathai Road, Pathumwan, Bangkok 10330, Thailand
}

(Received 22 February 2020; accepted 24 March 2020; published 10 April 2020)

\begin{abstract}
We study a number of supersymmetric solutions in the form of $M k w_{3} \times S^{3}$ - and $\mathrm{AdS}_{3} \times S^{3}$-sliced domain walls in the maximal gauged supergravity in seven dimensions. These solutions require nonvanishing three-form fluxes to support the $\mathrm{AdS}_{3}$ and $S^{3}$ subspaces. We consider solutions with $S O(4), S O(3), S O(2) \times S O(2)$, and $S O(2)$ symmetries in $C S O(p, q, 5-p-q), C S O(p, q, 4-p-q)$, and $S O(2,1) \ltimes \mathbf{R}^{4}$ gauge groups. All of these solutions can be analytically obtained. For $S O(5)$ and $\operatorname{CSO}(4,0,1)$ gauge groups, the complete truncation ansatze in terms of 11-dimensional supergravity on $S^{4}$ and type IIA theory on $S^{3}$ are known. We give the full uplifted solutions to 11 and 10 dimensions in this case. The solutions with an $\mathrm{AdS}_{3} \times S^{3}$ slice are interpreted as two-dimensional surface defects in sixdimensional $N=(2,0)$ superconformal field theory in the case of $S O(5)$ gauge group or $N=(2,0)$ nonconformal field theories for other gauge groups. For $S O(4)$ symmetric solutions, it is possible to find solutions with both the three-form fluxes and the $S O(3)$ gauge fields turned on. However, in this case, the solutions can be found only numerically. For $S O(3)$ symmetric solutions, the three-form fluxes and $S O(3)$ gauge fields cannot be nonvanishing simultaneously.
\end{abstract}

DOI: 10.1103/PhysRevD.101.086012

\section{INTRODUCTION}

Gauged supergravities in various spacetime dimensions have become a useful tool for studying different aspects of the AdS/CFT correspondence [1-3] and the DW/QFT correspondence [4-6]. Solutions to gauged supergravities provide some insight to the dynamics of strongly coupled conformal and nonconformal field theories via holographic descriptions; see, for example, [7-11]. The study along this line is particularly fruitful in the presence of supersymmetry. In this case, many aspects of both the gravity and the field theory sides are more controllable even at strong coupling. This makes finding various types of supersymmetric solutions in gauged supergravities worth considering.

In this paper, we are interested in supersymmetric solutions in the maximal gauged supergravity in seven dimensions. The solutions under consideration here take the form of $M k w_{3} \times S^{3}$ - and $\mathrm{AdS}_{3} \times S^{3}$-sliced domain walls. This type of solution has originally been considered in the minimal $N=2$ gauged supergravity in [12]; see also [13]

pparinya.ka@hotmail.com

†danai.nuchino@hotmail.com

Published by the American Physical Society under the terms of the Creative Commons Attribution 4.0 International license. Further distribution of this work must maintain attribution to the author(s) and the published article's title, journal citation, and DOI. Funded by SCOAP. for similar solutions in the matter-coupled $N=2$ gauged supergravity. Some of these solutions have been interpreted as surface defects within $N=(1,0)$ superconformal field theory (SCFT) in six dimensions in [14]; see [15,16] for similar solutions in six dimensions and [17-22] for examples of another holographic description of conformal defects in terms of Janus solutions.

We will find these $M k w_{3} \times S^{3}$ - and $\mathrm{AdS}_{3} \times S^{3}$-sliced domain walls in the maximal $N=4$ gauged supergravity with various types of gauge groups. The most general gaugings of the $N=4$ supergravity can be constructed by using the embedding tensor formalism [23]; for an earlier construction see $[24,25]$. The embedding tensor describes the embedding of an admissible gauge group $G_{0}$ in the global symmetry group $S L(5)$ and encodes all information about the resulting gauged supergravity. Supersymmetry allows for two components of the embedding tensor transforming in $\mathbf{1 5}$ and $\overline{\mathbf{4 0}}$ representations of $S L(5)$. We will consider $\operatorname{CSO}(p, q, 5-p-q)$ and $\operatorname{CSO}(p, q, 4-$ $p-q)$ gauge groups obtained from the embedding tensor in $\mathbf{1 5}$ and $\overline{\mathbf{4 0}}$ representations, respectively. We will also study similar solutions in $S O(2,1) \ltimes \mathbf{R}^{4}$ gauge group from the embedding tensor in both $\mathbf{1 5}$ and $\overline{\mathbf{4 0}}$ representations. Vacuum solutions in terms of half-supersymmetric domain walls for all these gauge groups have already been studied in [26]. In this paper, we will extend these solutions, which involve only the metric and scalars, by including nonvanishing two- and three-form fields. In some cases, in 
addition to two- and three-form fields, it is also possible to couple $S O(3)$ gauge fields to the solutions.

As shown in [27] using the framework of exceptional field theory, seven-dimensional gauged supergravity in $\mathbf{1 5}$ representation with $C S O(p, q, 5-p-q)$ gauge group can be obtained from a consistent truncation of 11-dimensional supergravity on $H^{p, q} \circ T^{5-p-q}$. On the other hand, a consistent truncation of type IIB theory on $H^{p, q} \circ T^{4-p-q}$ gives rise to $\operatorname{CSO}(p, q, 4-p-q)$ gauging from $\overline{\mathbf{4 0}}$ representation. This has been shown in [28] along with a partial result on the corresponding truncation ansatze. In particular, internal components of all 10-dimensional fields have been given.

For $S O(5)$ and $C S O(4,0,1)$ gauge groups, the complete truncation ansatze have already been constructed long ago in [29-31]. In this work, we will mainly consider uplifted solutions from these two gauge groups using the truncation ansatze given in [29-31], which are more useful for solutions involving two- and three-form fields in seven dimensions. We leave uplifting solutions from other gauge groups for future work.

The paper is organized as follows. In Sec. II, we give a brief review of the maximal gauged supergravity in seven dimensions. Supersymmetric $M k w_{3} \times S^{3}$ - and $\mathrm{AdS}_{3} \times S^{3}$ sliced domain walls in $\operatorname{CSO}(p, q, 5-p-q)$ gauge group together with the uplifted solutions to 11 and 10 dimensions in the case of $S O(5)$ and $C S O(4,0,1)$ gauge groups are presented in Sec. III. Similar solutions for $\operatorname{CSO}(p, q, 4-p-q)$ and $S O(2,1) \ltimes \mathbf{R}^{4}$ gauge groups obtained from gaugings in $\overline{\mathbf{4 0}}$ and $(\mathbf{1 5}, \overline{\mathbf{4 0}})$ representations are given in Secs. IV and V, respectively. Conclusions and comments are given in Sec. VI. In the two appendixes, all bosonic field equations of the maximal gauged supergravity and consistent truncation ansatze for 11-dimensional supergravity on $S^{4}$ and type IIA theory on $S^{3}$ are given.

\section{MAXIMAL GAUGED SUPERGRAVITY IN SEVEN DIMENSIONS}

In this section, we briefly review $N=4$ gauged supergravity in seven dimensions in the embedding tensor formalism. We mainly focus on the bosonic Lagrangian and fermionic supersymmetry transformations that are relevant for finding supersymmetric solutions. The reader is referred to [23] for the detailed construction of the maximal gauged supergravity.

As in other dimensions, the maximal $N=4$ supersymmetry in seven dimensions allows only the supergravity multiplet with the field content

$$
\left(e_{\mu}^{\hat{\mu}}, \psi_{\mu}^{a}, A_{\mu}^{M N}, B_{\mu \nu M}, \chi^{a b c}, \mathcal{V}_{M}^{A}\right)
$$

This multiplet consists of the graviton $e_{\mu}^{\hat{\mu}}$, four gravitini $\psi_{\mu}^{a}$, ten vectors $A_{\mu}^{M N}=A_{\mu}^{[M N]}$, five two-form fields $B_{\mu \nu M}, 16$ spin- $\frac{1}{2}$ fermions $\chi^{a b c}=\chi^{[a b] c}$, and 14 scalar fields described by the $S L(5) / S O(5)$ coset representative $\mathcal{V}_{M}{ }^{A}$.

Throughout the paper, we will use the following convention on various types of indices. Curved and flat spacetime indices are denoted by $\mu, \nu, \ldots$ and $\hat{\mu}, \hat{\nu}, \ldots$, respectively. Lower (upper) $M, N=1, \ldots, 5$ indices refer to the (anti)fundamental representation $5(\overline{\mathbf{5}})$ of the global $S L(5)$ symmetry. Accordingly, the vector $A_{\mu}^{M N}$ and twoform $B_{\mu \nu M}$ fields transform in the representations $\overline{\mathbf{1 0}}$ and $\mathbf{5}$, respectively.

On the other hand, fermionic fields transform in representations of the local $S O(5) \sim U S p(4)$ R-symmetry with $U S p(4)$ fundamental or $S O(5)$ spinor indices $a, b, \ldots=1$, $\ldots, 4$. The gravitini then transform as 4 while the spin- $\frac{1}{2}$ fields $\chi^{a b c}$ transform as $\mathbf{1 6}$ of $U S p(4)$. The latter satisfy the following conditions:

$$
\chi^{[a b c]}=0 \quad \text { and } \quad \Omega_{a b} \chi^{a b c}=0
$$

with $\Omega_{a b}=\Omega_{[a b]}$ being the $U S p(4)$ symplectic form satisfying the properties

$$
\left(\Omega_{a b}\right)^{*}=\Omega^{a b} \quad \text { and } \quad \Omega_{a c} \Omega^{b c}=\delta_{a}^{b} .
$$

It should also be noted that the raising and lowering of $U S p(4)$ indices by $\Omega^{a b}$ and $\Omega_{a b}$ correspond to complex conjugation. Furthermore, all fermions are symplectic Majorana spinors subject to the conditions

$$
\bar{\psi}_{\mu a}^{T}=\Omega_{a b} C \psi_{\mu}^{b} \quad \text { and } \quad \bar{\chi}_{a b c}^{T}=\Omega_{a d} \Omega_{b e} \Omega_{c f} C \chi^{\text {def }}
$$

where $C$ denotes the charge conjugation matrix obeying

$$
C=C^{T}=-C^{-1}=-C^{\dagger} .
$$

With the spacetime gamma matrices denoted by $\gamma^{\mu}$, the Dirac conjugate on a spinor $\Psi$ is defined by $\bar{\Psi}=\Psi^{\dagger} \gamma^{0}$.

The 14 scalars parametrizing $S L(5) / S O(5)$ coset are described by the coset representative $\mathcal{V}_{M}{ }^{A}$, transforming under the global $S L(5)$ and local $S O(5)$ symmetries by left and right multiplications. Indices $M=1,2, \ldots, 5$ and $A=$ $1,2, \ldots, 5$ are accordingly $S L(5)$ and $S O(5)$ fundamental indices, respectively. To couple fermions that transform under $U S p(4)$, we write the $S O(5)$ vector indices of $\mathcal{V}_{M}{ }^{A}$ as a pair of antisymmetric $U S p(4)$ fundamental indices in the form of $\mathcal{V}_{M}{ }^{a b}=\mathcal{V}_{M}{ }^{[a b]}$. In addition, the coset representative $\mathcal{V}_{M}{ }^{a b}$ satisfies the relation

$$
\mathcal{V}_{M}{ }^{a b} \Omega_{a b}=0 .
$$

Similarly, the inverse of $\mathcal{V}_{M}{ }^{A}$ denoted by $\mathcal{V}_{A}{ }^{M}$ will be written as $\mathcal{V}_{a b}{ }^{M}$. We then have the following relations:

$\mathcal{V}_{M}{ }^{a b} \mathcal{V}_{a b}{ }^{N}=\delta_{M}^{N} \quad$ and $\quad \mathcal{V}_{a b}{ }^{M} \mathcal{V}_{M}{ }^{c d}=\delta_{a}^{[c} \delta_{b}^{d]}-\frac{1}{4} \Omega_{a b} \Omega^{c d}$ 
Gaugings are deformations of the $N=4$ supergravity by promoting a subgroup $G_{0} \subset S L(5)$ to be a local symmetry. The most general gaugings of a supergravity theory can be efficiently described by using the embedding tensor formalism. The embedding of $G_{0}$ within $S L(5)$ is achieved by using a constant $S L(5)$ tensor $\Theta_{M N, P} Q=\Theta_{[M N], P} Q$ living in the product representation [23]

$$
10 \otimes 24=10+15+\overline{40}+175 .
$$

It turns out that supersymmetry allows only the embedding tensor in the $\mathbf{1 5}$ and $\overline{\mathbf{4 0}}$ representations. These two representations can be described by the tensors $Y_{M N}$ and $Z^{M N, P}$ with $Y_{M N}=Y_{(M N)}, Z^{M N, P}=Z^{[M N], P}$, and $Z^{[M N, P]}=0$ in terms of which the embedding tensor can be written as

$$
\Theta_{M N, P} Q=\delta_{[M}^{Q} Y_{N] P}-2 \epsilon_{M N P R S} Z^{R S, Q} .
$$

In term of the embedding tensor, gauge generators are given by

$$
X_{M N}=\Theta_{M N, P} Q_{t}^{P}{ }_{Q}
$$

in which $t^{M}{ }_{N}$, satisfying $t^{M}{ }_{M}=0$, are $S L(5)$ generators. In particular, the gauge generators in the fundamental $\mathbf{5}$ and $\mathbf{1 0}$ representations are given by

$$
\begin{gathered}
X_{M N, P} Q=\Theta_{M N, P} Q=\delta_{[M}^{Q} Y_{N] P}-2 \epsilon_{M N P R S} Z^{R S, Q}, \\
\text { and } \quad\left(X_{M N}\right)_{P Q}{ }^{R S}=2 X_{M N,[P}{ }^{[R} \delta_{Q]}^{S]}
\end{gathered}
$$

with $\epsilon_{M N P Q R}$ being the invariant tensor of $S L(5)$. To ensure that the gauge generators form a closed subalgebra of $S L(5)$

$$
\left[X_{M N}, X_{P Q}\right]=-\left(X_{M N}\right)_{P Q}{ }^{R S} X_{R S},
$$

the embedding tensor needs to satisfy the quadratic constraint

$$
Y_{M Q} Z^{Q N, P}+2 \epsilon_{M R S T U} Z^{R S, N} Z^{T U, P}=0 .
$$

Gaugings introduce minimal coupling between the gauge fields and other fields via the covariant derivative

$$
D_{\mu}=\nabla_{\mu}-g A_{\mu}^{M N} \Theta_{M N, P} Q_{t}^{P}{ }_{Q},
$$

where $\nabla_{\mu}$ is the spacetime covariant derivative including (possibly) composite $S O(5)$ connections. To restore supersymmetry of the original $N=4$ supergravity, fermionic masslike terms and the scalar potential at first and second orders in the gauge coupling constant are needed. In addition, to ensure gauge covariance, the field strength tensors of vector and two-form fields need to be modified as

$$
\begin{gathered}
\mathcal{H}_{\mu \nu}^{(2) M N}=F_{\mu \nu}^{M N}+g Z^{M N, P} B_{\mu \nu P}, \\
\mathcal{H}_{\mu \nu \rho M}^{(3)}=g Y_{M N} S_{\mu \nu \rho}^{N}+3 D_{[\mu} B_{\nu \rho] M} \\
+6 \epsilon_{M N P Q R} A_{[\mu}^{N P}\left(\partial_{\nu} A_{\rho]}^{Q R}+\frac{2}{3} g X_{S T, U}{ }^{Q} A_{\nu}^{R U} A_{\rho]}^{S T}\right),
\end{gathered}
$$

where the non-Abelian gauge field strength tensor is defined by

$$
F_{\mu \nu}^{M N}=2 \partial_{[\mu} A_{\nu]}^{M N}+g\left(X_{P Q}\right)_{R S}{ }^{M N} A_{[\mu}^{P Q} A_{\nu]}^{R S} .
$$

Note that the three-form fields $S_{\mu \nu \rho}^{M}$ in $H_{\mu \nu \rho}^{(3)}$ only appear under the projection of $Y_{M N}$. In ungauged supergravity, all of the three-form fields can be dualized to two-form fields. However, this is not the case in the gauged supergravity. Therefore, different gaugings lead to different field contents in the resulting gauged supergravity.

Following [23], we first define $s \equiv \operatorname{rank} Z$ and $t \equiv \operatorname{rank} Y$. In a given gauging, $t$ two-forms can be set to zero by tensor gauge transformations of the three-form fields. This results in $t$ self-dual massive three-forms. Similarly, $s$ gauge fields can be set to zero by tensor gauge transformations of the two-forms giving rise to $s$ massive two-form fields. It should also be pointed out that there can be massive vector fields arising from broken gauge symmetry via the usual Higgs mechanism. We can see that the numbers of two- and three-form tensor fields depend on the gauging under consideration. However, the quadratic constraint ensures that $t+s \leq 5$, so the degrees of freedom from the ten vector and five two-form fields in the ungauged supergravity are redistributed into two- and three-form fields in the gauged theory. This fact will affect our ansatz for finding supersymmetric solutions in subsequent sections. To summarize, we repeat the distribution of degrees of freedom after gauge fixing from [23] in Table I.

The covariance two- and three-form field strengths satisfy the following modified Bianchi identities:

$$
\begin{gathered}
D_{[\mu} \mathcal{H}_{\nu \rho]}^{(2) M N}=\frac{1}{3} g Z^{M N, P} \mathcal{H}_{\mu \nu \rho P}^{(3)}, \\
D_{[\mu} \mathcal{H}_{\nu \rho \lambda] M}^{(3)}=\frac{3}{2} \epsilon_{M N P Q R} \mathcal{H}_{[\mu \nu}^{(2) N P} \mathcal{H}_{\rho \lambda]}^{(2) Q R}+\frac{1}{4} g Y_{M N} \mathcal{H}_{\mu \nu \rho \lambda}^{(4) N},
\end{gathered}
$$

TABLE I. Distribution of the tensor fields' degrees of freedom after gauge fixing.

\begin{tabular}{lcc}
\hline \hline Fields & $\#$ & \# d.o.f \\
\hline Massless vectors & $10-s$ & 5 \\
Massless 2-forms & $5-s-t$ & 10 \\
Massive 2-forms & $s$ & 15 \\
Massive sd. 3-forms & $t$ & 10 \\
\hline \hline
\end{tabular}


where the covariant field strengths of the three-form fields are given by

$$
\begin{aligned}
Y_{M N} \mathcal{H}_{\mu \nu \rho \lambda}^{(4) N}= & Y_{M N}\left[4 D_{[\mu} S_{\nu \rho \lambda]}^{N}+6 F_{[\mu \nu}^{N P} B_{\rho \lambda] P}+3 g Z^{N P, Q} B_{[\mu \nu P} B_{\rho \lambda] Q}\right. \\
& \left.+4 g \epsilon_{P Q R V W} X_{S T, U}{ }^{N} A_{[\mu}^{N P} A_{\nu}^{Q R} A_{\rho}^{S T} A_{\lambda]}^{U W}+8 \epsilon_{P Q R S T} A_{[\mu}^{N P} A_{\nu}^{Q R} \partial_{\rho} A_{\lambda]}^{S T}\right] .
\end{aligned}
$$

It should be emphasized that the three-forms $S_{\mu \nu \rho}^{M}$ and its field strength tensors always appear under the projection by $Y_{M N}$.

With all these ingredients, the bosonic Lagrangian of the seven-dimensional maximal gauged supergravity can be written as

$$
\begin{aligned}
e^{-1} \mathcal{L}= & \frac{1}{2} R-\mathcal{M}_{M P} \mathcal{M}_{N Q} \mathcal{H}_{\mu \nu}^{(2) M N} \mathcal{H}^{(2) P Q \mu \nu}-\frac{1}{6} \mathcal{M}^{M N} \mathcal{H}_{\mu \nu \rho M}^{(3)} \mathcal{H}^{(3) \mu \nu \rho}{ }_{N} \\
& +\frac{1}{8}\left(D_{\mu} \mathcal{M}_{M N}\right)\left(D^{\mu} \mathcal{M}^{M N}\right)-e^{-1} \mathcal{L}_{V T}-\mathbf{V} .
\end{aligned}
$$

In this equation, the scalar fields are described by a unimodular symmetric matrix

$$
\mathcal{M}_{M N}=\mathcal{V}_{M}{ }^{a b} \mathcal{V}_{N}{ }^{c d} \Omega_{a c} \Omega_{b d}
$$

Its inverse is given by

$$
\mathcal{M}^{M N}=\mathcal{V}_{a b}{ }^{M} \mathcal{V}_{c d}{ }^{N} \Omega^{a c} \Omega^{b d}
$$

We will not give the explicit form of the vector-tensor topological term $\mathcal{L}_{V T}$ here due to its complexity but refer the reader to [23]. Finally, the scalar potential is given by

$$
\begin{aligned}
\mathbf{V} & =\frac{g^{2}}{64}\left[2 \mathcal{M}^{M N} Y_{N P} \mathcal{M}^{P Q} Y_{Q M}-\left(\mathcal{M}^{M N} Y_{M N}\right)^{2}\right] \\
& +g^{2} Z^{M N, P} Z^{Q R, S}\left(\mathcal{M}_{M Q} \mathcal{M}_{N R} \mathcal{M}_{P S}-\mathcal{M}_{M Q} \mathcal{M}_{N P} \mathcal{M}_{R S}\right)
\end{aligned}
$$

The supersymmetry transformations of fermionic fields that are essential for finding supersymmetric solutions read

$$
\begin{aligned}
\delta \psi_{\mu}^{a}= & D_{\mu} \epsilon^{a}-g \gamma_{\mu} A_{1}^{a b} \Omega_{b c} \epsilon^{c}+\frac{1}{15} \mathcal{H}_{\nu \rho \lambda M}^{(3)}\left(\gamma_{\mu}{ }^{\nu \rho \lambda}-\frac{9}{2} \delta_{\mu}^{\nu} \gamma^{\rho \lambda}\right) \Omega^{a b} \mathcal{V}_{b c}{ }^{M} \epsilon^{c} \\
& +\frac{1}{5} \mathcal{H}_{\nu \rho}^{(2) M N}\left(\gamma_{\mu}{ }^{\nu \rho}-8 \delta_{\mu}^{\nu} \gamma^{\rho}\right) \mathcal{V}_{M}{ }^{a d} \Omega_{d e} \mathcal{V}_{N}{ }^{e b} \Omega_{b c} \epsilon^{c}, \\
\delta \chi^{a b c}= & 2 \Omega^{c d} P_{\mu d e}{ }^{a b} \gamma^{\mu} \epsilon^{e}+g A_{2}^{d, a b c} \Omega_{d e} \epsilon^{e} \\
+ & 2 \mathcal{H}_{\mu \nu}^{(2) M N} \gamma^{\mu \nu} \Omega_{d e}\left[\mathcal{V}_{M}{ }^{c d} \mathcal{V}_{N}{ }^{e[a} \epsilon^{b]}-\frac{1}{5}\left(\Omega^{a b} \delta_{g}^{c}-\Omega^{c[a} \delta_{g}^{b]}\right) \mathcal{V}_{M^{g f}} \Omega_{f h} \mathcal{V}_{N}{ }^{h d} \epsilon^{e}\right] \\
& -\frac{1}{6} \mathcal{H}_{\mu \nu \rho M}^{(3)} \gamma^{\mu \nu \rho} \mathcal{V}_{f e}{ }^{M}\left[\Omega^{a f} \Omega^{b e} \epsilon^{c}-\frac{1}{5}\left(\Omega^{a b} \Omega^{c f}+4 \Omega^{c[a} \Omega^{b] f}\right) \epsilon^{e}\right] .
\end{aligned}
$$

The covariant derivative of the supersymmetry parameters is defined by

$$
D_{\mu} \epsilon^{a}=\nabla_{\mu} \epsilon^{a}-Q_{\mu b}{ }^{a} \epsilon^{b} .
$$

The composite connection $Q_{\mu a}{ }^{b}$ and the vielbein on the $S L(5) / S O(5) \operatorname{coset} P_{\mu a b}{ }^{c d}$ are obtained from the following relation:

$$
P_{\mu a b}{ }^{c d}+2 Q_{\mu[a}{ }^{[c} \delta_{b]}^{d]}=\mathcal{V}_{a b}{ }^{M}\left(\partial_{\mu} \mathcal{V}_{M}{ }^{c d}-g A_{\mu}^{P Q} X_{P Q, M}{ }^{N} \mathcal{V}_{N}{ }^{c d}\right)
$$


The fermion shift matrices $A_{1}$ and $A_{2}$ are given by

$$
\begin{gathered}
A_{1}^{a b}=-\frac{1}{4 \sqrt{2}}\left(\frac{1}{4} B \Omega^{a b}+\frac{1}{5} C^{a b}\right), \\
A_{2}^{d, a b c}=\frac{1}{2 \sqrt{2}}\left[\Omega^{e c} \Omega^{f d}\left(C^{a b}{ }_{e f}-B^{a b}{ }_{e f}\right)\right. \\
\left.+\frac{1}{4}\left(C^{a b} \Omega^{c d}+\frac{1}{5} \Omega^{a b} C^{c d}+\frac{4}{5} \Omega^{c[a} C^{b] d}\right)\right]
\end{gathered}
$$

with various components of $B$ and $C$ tensors defined by

$$
\begin{gathered}
B=\frac{\sqrt{2}}{5} \Omega^{a c} \Omega^{b d} Y_{a b, c d} \\
B^{a b}{ }_{c d}=\sqrt{2}\left[\Omega^{a e} \Omega^{b f} \delta_{c}^{[g} \delta_{d}^{h]}\right. \\
\left.-\frac{1}{5}\left(\delta_{c}^{[a} \delta_{d}^{b]}-\frac{1}{4} \Omega^{a b} \Omega_{c d}\right) \Omega^{e g} \Omega^{f h}\right] Y_{e f, g h}, \\
C^{a b}=8 \Omega_{c d} Z^{(a c)[b d]}, \\
C^{a b}{ }_{c d}=8\left(-\Omega_{c e} \Omega_{d f} \delta_{g}^{[a} \delta_{h}^{b]}+\Omega_{g(c} \delta_{d d)}^{[a} \delta_{e}^{b]} \Omega_{f h}\right) Z^{(e f)[g h]} .
\end{gathered}
$$

In the above equations, we have introduced "dressed" components of the embedding tensor defined by

$$
Y_{a b, c d}=\mathcal{V}_{a b}{ }^{M} \mathcal{V}_{c d}{ }^{N} Y_{M N}
$$

and $\quad Z^{(a c)[e f]}=\sqrt{2} \mathcal{V}_{M}^{a b} \mathcal{V}_{N}^{c d} \mathcal{V}_{P}^{e f} \Omega_{b d} Z^{M N, P}$.

Finally, we note that the scalar potential can also be written in terms of the fermion-shift matrices $A_{1}$ and $A_{2}$ as $\mathbf{V}=-15 A_{1}^{a b} A_{1 a b}+\frac{1}{8} A_{2}^{a, b c d} A_{2 a, b c d}=-15\left|A_{1}\right|^{2}+\frac{1}{8}\left|A_{2}\right|^{2}$.

In the following sections, we will find supersymmetric solutions in a number of possible gauge groups.

\section{SUPERSYMMETRIC SOLUTIONS FROM GAUGINGS IN 15 REPRESENTATION}

We begin with gaugings in $\mathbf{1 5}$ representation with $Z^{M N, P}=0$. The $S L(5)$ symmetry can be used to bring $Y_{M N}$ to the form

$$
\begin{aligned}
Y_{M N}= & \operatorname{diag}(\underbrace{1, \ldots, 1}_{p}, \underbrace{-1, \ldots,-1}_{q}, \underbrace{0, \ldots, 0}_{r}), \\
& p+q+r=5 .
\end{aligned}
$$

This corresponds to the gauge group

$$
C S O(p, q, r) \sim S O(p, q) \ltimes \mathbf{R}^{(p+q) r} .
$$

To give an explicit parametrization of the $S L(5) / S O(5)$ coset, we first introduce $G L(5)$ matrices

$$
\left(e_{M N}\right)_{K}{ }^{L}=\delta_{M K} \delta_{N}^{L} .
$$

We will use the following choice of $S O(5)$ gamma matrices to convert an $S O(5)$ vector index to a pair of antisymmetric spinor indices

$$
\begin{aligned}
& \Gamma_{1}=-\sigma_{2} \otimes \sigma_{2}, \quad \Gamma_{2}=\mathbf{I}_{2} \otimes \sigma_{1}, \quad \Gamma_{3}=\mathbf{I}_{2} \otimes \sigma_{3}, \\
& \Gamma_{4}=\sigma_{1} \otimes \sigma_{2},
\end{aligned}
$$

where $\sigma_{i}$ are the usual Pauli matrices. $\Gamma_{A}$ satisfy the following relations:

$$
\begin{aligned}
\left\{\Gamma_{A}, \Gamma_{B}\right\} & =2 \delta_{A B} \mathbf{I}_{4}, \quad\left(\Gamma_{A}\right)^{a b}=-\left(\Gamma_{A}\right)^{b a}, \\
\Omega_{a b}\left(\Gamma_{A}\right)^{a b} & =0, \quad\left(\left(\Gamma_{A}\right)^{a b}\right)^{*}=\Omega_{a c} \Omega_{b d}\left(\Gamma_{A}\right)^{c d} .
\end{aligned}
$$

The symplectic form of $U S p(4)$ is chosen to be

$$
\Omega_{a b}=\Omega^{a b}=\mathbf{I}_{2} \otimes i \sigma_{2} .
$$

The coset representative of the form $\mathcal{V}_{M}{ }^{a b}$ and the inverse $\mathcal{V}_{a b}{ }^{M}$ are then obtained from the following relations:

$\mathcal{V}_{M}{ }^{a b}=\frac{1}{2} \mathcal{V}_{M}{ }^{A}\left(\Gamma_{A}\right)^{a b} \quad$ and $\quad \mathcal{V}_{a b}{ }^{M}=\frac{1}{2} \mathcal{V}_{A}{ }^{M}\left(\Gamma^{A}\right)_{a b}$

We will use the metric ansatz in the form of an $\mathrm{AdS}_{3} \times S^{3}$-sliced domain wall

$$
d s_{7}^{2}=e^{2 U(r)} d s_{\mathrm{AdS}_{3}}^{2}+e^{2 V(r)} d r^{2}+e^{2 W(r)} d s_{S^{3}}^{2} .
$$

The seven-dimensional coordinates are taken to be $x^{\mu}=$ $\left(x^{m}, r, x^{i}\right)$ with $m=0,1,2$ and $i=4,5,6$. Note that $V(r)$ is an arbitrary nondynamical function that can be set to zero with a suitable gauge choice. The explicit forms for the metrics on $\mathrm{AdS}_{3}$ and $S^{3}$ are given in Hopf coordinates by

$$
d s_{\mathrm{AdS}_{3}}^{2}=\frac{1}{\tau^{2}}\left[-d t^{2}+\left(d x^{1}\right)^{2}+\left(d x^{2}\right)^{2}+2 \sinh x^{1} d t d x^{2}\right],
$$

$d s_{S^{3}}^{2}=\frac{1}{\kappa^{2}}\left[\left(d x^{4}\right)^{2}+\left(d x^{5}\right)^{2}+\left(d x^{6}\right)^{2}+2 \sin x^{5} d x^{4} d x^{6}\right]$,

in which $\tau$ and $\kappa$ are constants. In the limit $\tau \rightarrow 0$ and $\kappa \rightarrow 0$, the $\mathrm{AdS}_{3}$ and $S^{3}$ parts become flat Minkowski space $M k w_{3}$ and flat space $\mathbb{R}^{3}$, respectively. 
With the following choice of vielbeins:

$$
\begin{aligned}
e^{\hat{0}} & =\frac{1}{\tau} e^{U(r)}\left(d t-\sinh x^{1} d x^{2}\right), \quad e^{\hat{1}}=\frac{1}{\tau} e^{U(r)}\left(\cos t d x^{1}-\sin t \cosh x^{1} d x^{2}\right), \\
e^{\hat{2}} & =\frac{1}{\tau} e^{U(r)}\left(\sin t d x^{1}+\cos t \cosh x^{1} d x^{2}\right), \quad e^{\hat{3}}=e^{V(r)} d r, \\
e^{\hat{4}} & =\frac{1}{\kappa} e^{W(r)}\left(d x^{4}+\sin x^{5} d x^{6}\right), \quad e^{\hat{5}}=\frac{1}{\kappa} e^{W(r)}\left(\cos x^{4} d x^{5}-\sin x^{4} \cos x^{5} d x^{6}\right), \\
e^{\hat{6}} & =\frac{1}{\kappa} e^{W(r)}\left(\sin x^{4} d x^{5}+\cos x^{4} \cos x^{5} d x^{6}\right),
\end{aligned}
$$

we find the following nonvanishing components of the spin connection:

$$
\begin{aligned}
\omega_{\hat{n}}^{\hat{m}} \hat{\hat{3}}=e^{-V(r)} U^{\prime}(r) \delta_{\hat{n}}^{\hat{m}}, & \omega_{\hat{m} \hat{n} \hat{p}}=\frac{\tau}{2} e^{-U(r)} \varepsilon_{\hat{m} \hat{n} \hat{p}}, \\
\omega_{\hat{j} \hat{\jmath}}^{\hat{i}}=e^{-V(r)} W^{\prime}(r) \delta_{\hat{j}}^{\hat{i}}, & \omega_{\hat{i} \hat{j} \hat{k}}=\frac{\kappa}{2} e^{-W(r)} \varepsilon_{\hat{i} \hat{j} \hat{k}}
\end{aligned}
$$

with the convention that $\varepsilon_{\hat{0} \hat{1} \hat{2}}=-\varepsilon^{\hat{0} \hat{1} \hat{2}}=\varepsilon_{\hat{4} \hat{5} \hat{\sigma}}=\varepsilon^{\hat{4} \hat{5} \hat{b}}=1$. Throughout this paper, we will use a prime to denote the $r$ derivative.

Following [12], we take the ansatz for the Killing spinors to be

$$
\epsilon^{a}=e^{U(r) / 2}\left[\cos \theta(r) \mathbf{I}_{8}+\sin \theta(r) \gamma^{\hat{0} \hat{1} \hat{2}}\right] \epsilon_{0}^{a}
$$

with $\epsilon_{0}^{a}$ being constant spinors. In addition, we will use the following ansatz for the three-form field strength tensors:

$$
\begin{aligned}
\mathcal{H}_{\hat{m} \hat{n} \hat{p} M}^{(3)} & =k_{M}(r) e^{-3 U(r)} \varepsilon_{\hat{m} \hat{n} \hat{p}} \quad \text { and } \\
\mathcal{H}_{\hat{i} \hat{j} \hat{k} M}^{(3)} & =l_{M}(r) e^{-3 W(r)} \varepsilon_{\hat{i} \hat{j} \hat{k}}
\end{aligned}
$$

or, equivalently,

$$
\mathcal{H}_{M}^{(3)}=k_{M} \operatorname{vol}_{\mathrm{AdS}_{3}}+l_{M} \operatorname{vol}_{S^{3}} .
$$

In subsequent analysis, we will call the solutions with nonvanishing $\mathcal{H}_{M}^{(3)}$ "charged" domain walls.

\section{A. $S O(4)$ symmetric charged domain walls}

We first consider charged domain wall solutions with $S O$ (4) symmetry. As in [26], we will find supersymmetric solutions with a given unbroken symmetry from many gauge groups within a single framework. Gauge groups that can give rise to $S O(4)$ symmetric solutions are $S O(5)$, $S O(4,1)$, and $C S O(4,0,1)$. We will accordingly write $Y_{M N}$ in the following form:

$$
Y_{M N}=\operatorname{diag}(+1,+1,+1,+1, \rho),
$$

where $\rho=+1,-1,0$ corresponding to $S O(5), S O(4,1)$, and $\operatorname{CSO}(4,0,1)$ gauge groups, respectively. With this embedding tensor, the $S O(4)$ residual symmetry is generated by $X_{M N}$ with $M, N=1,2,3,4$.

Among the 14 scalars in $S L(5) / S O(5)$ coset, there is one $S O(4)$ invariant scalar corresponding to the noncompact generator

$$
\hat{Y}=e_{1,1}+e_{2,2}+e_{3,3}+e_{4,4}-4 e_{5,5} .
$$

With the coset representative

$$
\mathcal{V}=e^{\phi \hat{Y}},
$$

the scalar potential is given by

$$
\mathbf{V}=-\frac{g^{2}}{64} e^{-4 \phi}\left(8+8 \rho e^{10 \phi}-\rho^{2} e^{20 \phi}\right) .
$$

For $\rho=1$, this potential admits two $\mathrm{AdS}_{7}$ critical points with $S O(5)$ and $S O(4)$ unbroken symmetries. The former preserves all supersymmetry while the latter is nonsupersymmetric. These vacua are given, respectively, by

$$
\phi=0 \quad \text { and } \quad \mathbf{V}_{0}=-\frac{15}{64} g^{2}
$$

and

$$
\phi=\frac{1}{10} \ln 2 \quad \text { and } \quad \mathbf{V}_{0}=-\frac{5 g^{2}}{16 \times 2^{2 / 5}} .
$$

The cosmological constant is denoted by $\mathbf{V}_{0}$, the value of the scalar potential at the vacuum.

To preserve $S O(4)$ symmetry, we will keep only the following components of $\mathcal{H}_{M}^{(3)}$ nonvanishing

$$
\begin{gathered}
\mathcal{H}_{\hat{m} \hat{n} \hat{p} 5}^{(3)}=k(r) e^{-3 U(r)} \varepsilon_{\hat{m} \hat{n} \hat{p}} \quad \text { and } \\
\mathcal{H}_{\hat{i} \hat{j} \hat{k} 5}^{(3)}=l(r) e^{-3 W(r)} \varepsilon_{\hat{i} \hat{j} \hat{k}} .
\end{gathered}
$$

At this point, it is useful to consider the $\mathcal{H}_{M}^{(3)}$ contribution in more detail. For $S O(5)$ and $S O(4,1)$ gauge groups corresponding to a nondegenerate $Y_{M N}$, the field content of the gauged supergravity contains $t=5$ massive three-form 
fields $S_{\mu \nu \rho}^{M}$. For vanishing gauge and two-form fields, the field strength tensor $\mathcal{H}_{M}^{(3)}$ is then given by

$$
\mathcal{H}_{\mu \nu \rho M}^{(3)}=g Y_{M N} S_{\mu \nu \rho}^{N} .
$$

Since the four-form field strengths do not enter the supersymmetry transformations of fermionic fields, the functions $k_{M}(r)$ and $l_{M}(r)$ will appear, in this case, algebraically in the resulting Bogomol'nyi-PrasadSommereld (BPS) equations. This is in contrast to the pure $N=2$ gauged supergravity considered in [12] in which the four-form field strength of the massive threeform field appears in the supersymmetry transformations. Therefore, in that case, the BPS conditions result in differential equations for $k(r)$ and $l(r)$.

For $\operatorname{CSO}(4,0,1)$ gauge group with $Y_{55}=0, S_{\mu \nu \rho}^{5}$ does not contribute to $\mathcal{H}_{M}^{(3)}$, but, in this case with $s=0$ and $t=4$, there is $5-t=1$ massless two-form field $B_{\mu \nu 5}$ with the field strength

$$
\mathcal{H}_{\mu \nu \rho 5}^{(3)}=3 D_{[\mu} B_{\nu \rho] 5} .
$$

To satisfy Bianchi's identity $D \mathcal{H}^{(3)}=0$, we need $k^{\prime}=l^{\prime}=$ 0 or constant three-form fluxes. We will see that this is indeed the case for our BPS solutions. Taking this condition into account, we can write the ansatz for the twoform field as

$$
B_{M}=k_{M}(r) \omega_{2}+l_{M}(r) \tilde{\omega}_{2}
$$

with $\operatorname{vol}_{\mathrm{AdS}_{3}}=d \omega_{2}$ and $\operatorname{vol}_{S^{3}}=d \tilde{\omega}_{2}$. With the metrics given in (47) and (48), the explicit form of $\omega_{2}$ and $\tilde{\omega}_{2}$ is given by

$$
\begin{aligned}
& \omega_{2}=-\frac{1}{\tau^{3}} \sinh x^{1} d t \wedge d x^{2} \quad \text { and } \\
& \tilde{\omega}_{2}=-\frac{1}{\kappa^{3}} \sin x^{5} d x^{4} \wedge d x^{6} .
\end{aligned}
$$

After imposing two projection conditions

$$
\gamma_{\hat{3}} \epsilon_{0}^{a}=\left(\Gamma_{5}\right)^{a}{ }_{b} \epsilon_{0}^{b}=\epsilon_{0}^{a},
$$

we find the following BPS equations from the conditions $\delta \psi_{\mu}^{a}=0$ and $\delta \chi^{a b c}=0$ :

$$
\begin{aligned}
U^{\prime}= & \frac{e^{V-2 \phi}}{80 \cos 2 \theta}\left[g\left(8-\rho e^{10 \phi}\right)+3 g \rho e^{10 \phi} \cos 4 \theta\right. \\
& \left.-16 \tau e^{2 \phi-U} \sin 2 \theta\right], \\
W^{\prime}= & \frac{e^{V-2 \phi}}{40 \cos 2 \theta}\left[g\left(4+2 \rho e^{10 \phi}\right)-g \rho e^{10 \phi} \cos 4 \theta\right. \\
& \left.-8 \tau e^{2 \phi-U} \sin 2 \theta\right],
\end{aligned}
$$

$$
\begin{gathered}
\phi^{\prime}=\frac{e^{V-2 \phi}}{80 \cos 2 \theta}\left[g\left(4-3 \rho e^{10 \phi}\right)-g \rho e^{10 \phi} \cos 4 \theta\right. \\
\left.-8 \tau e^{2 \phi-U} \sin 2 \theta\right], \\
\theta^{\prime}=-\frac{1}{16} g \rho e^{V+8 \phi} \sin 2 \theta, \\
k=\frac{1}{8} e^{2 U-4 \phi}\left(4 \tau-g \rho e^{U+8 \phi} \sin 2 \theta\right), \\
l=\frac{1}{8} e^{3 W-6 \phi}\left[g\left(\rho e^{10 \phi}-2\right) \tan 2 \theta+4 \tau e^{2 \phi-U} \sec 2 \theta\right],
\end{gathered}
$$

together with an algebraic constraint

$$
0=e^{-W} \kappa-e^{-U} \tau \sec 2 \theta+\frac{1}{2} g e^{-2 \phi} \tan 2 \theta .
$$

We note here that the appearance of the $S O(5)$ gamma matrix $\Gamma_{5}$ in the projection conditions is due to the nonvanishing $\mathcal{H}_{\mu \nu \rho 5}^{(3)}$. Note also that the solutions are $\frac{1}{4}$ BPS since the Killing spinors $\epsilon_{0}^{a}$ are subject to two projectors. We now consider various possible solutions to these BPS equations.

\section{1. $M k w_{3} \times \mathbb{R}^{3}$-sliced domain walls}

We begin with a simple case of $M k w_{3} \times \mathbb{R}^{3}$-sliced domain walls with vanishing $\tau$ and $\kappa$. Imposing $\tau=\kappa=$ 0 into the constraint (72) gives

$$
0=\frac{1}{2} g e^{-2 \phi} \tan 2 \theta
$$

Setting $g=0$ corresponds to ungauged $N=4$ supergravity and gives rise to a supersymmetric $M k w_{3} \times \mathbb{R} \times \mathbb{R}^{3} \sim$ $M k w_{7}$ background as expected.

Another possibility to satisfy the condition (73) is to set $\tan 2 \theta=0$, which implies $\theta=\frac{n \pi}{2}, n=0,1,2,3, \ldots$. For even $n$, we have $\sin \theta=0$, and from (51), the Killing spinors take the form

$$
\epsilon^{a}=e^{U(r) / 2} \epsilon_{0}^{a}
$$

with $\epsilon_{0}^{a}$ satisfying the projection conditions given in (65). For odd $n$ with $\cos \theta=0$, the Killing spinors become

$$
\epsilon^{a}=e^{U(r) / 2} \gamma^{\hat{0} \hat{1} \hat{2}} \epsilon_{0}^{a}
$$

We can redefine $\epsilon_{0}^{a}$ to $\tilde{\epsilon}_{0}^{a}=\gamma^{\hat{0} \hat{1} \hat{2}} \epsilon_{0}^{a}$ satisfying the projection conditions

$$
-\gamma_{\hat{3}} \epsilon_{0}^{a}=\left(\Gamma_{5}\right)^{a}{ }_{b} \epsilon_{0}^{b}=\epsilon_{0}^{a} .
$$

This differs from the projectors in (65) only by a minus sign in the $\gamma_{\hat{3}}$ projector. Therefore, the two possibilities obtained 
from the condition $\tan 2 \theta=0$ are equivalent by flipping the sign of the $\gamma_{\hat{3}}$ projector. We can accordingly choose $\theta=0$ without losing any generality.

With $\theta=0$, the BPS equations (66) to (71) become

$$
\begin{gathered}
U^{\prime}=W^{\prime}=\frac{1}{40} g e^{V-2 \phi}\left(4+\rho e^{10 \phi}\right), \\
\phi^{\prime}=\frac{1}{20} g e^{V-2 \phi}\left(1-\rho e^{10 \phi}\right), \\
k=l=0 .
\end{gathered}
$$

By choosing $V=-3 \phi$, we find the following solution:

$$
\begin{gathered}
U=W=2 \phi-\frac{1}{4} \ln \left[1-\rho e^{10 \phi}\right], \\
e^{5 \phi}=\frac{1}{\sqrt{\rho}} \tanh \left[\frac{\sqrt{\rho}}{4}(g r+C)\right]
\end{gathered}
$$

with an integration constant $C$. Since $k=l=\theta=0$, the $\Gamma_{5}$ projection in (65) is not needed. This is then a halfsupersymmetric solution with vanishing three-form fluxes and is exactly the $S O(4)$ symmetric domain wall studied in [26]. Therefore, the $M k w_{3} \times \mathbb{R}^{3}$-sliced solution is just the standard flat domain wall.

\section{2. $M k w_{3} \times S^{3}$-sliced domain walls}

In this case, we look for domain wall solutions with $M k w_{3} \times S^{3}$ slice. Following [12], we choose the following gauge choice:

$$
e^{-V}=\frac{1}{16} e^{8 \phi}
$$

By setting $\tau=0$, we can solve the BPS equations (66)-(71) and obtain the following solution for $\rho= \pm 1$ :

$$
\begin{gathered}
U=2 \phi-\ln (\sin 2 \theta), \\
W=2 \phi-\ln (\tan 2 \theta), \\
e^{10 \phi}=2 C(\cos 4 \theta-3)+(4 C+\rho) \sec ^{2} 2 \theta, \\
k=-\frac{g}{8}\left(4 \rho C+\csc ^{4} 2 \theta\right) \tan ^{2} 2 \theta, \\
l=\frac{g}{16}[\rho C(\cos 8 \theta+3)-2(2 \rho C+1) \cos 4 \theta] \csc ^{2} 2 \theta, \\
\theta=\arctan \left(e^{-2 g \rho r}\right)
\end{gathered}
$$

with $\kappa=-g / 2$. $C$ is an integration constant in the solution for $\phi$.
For $S O(5)$ gauge group with $\rho=1$, the solution is locally asymptotic to the $N=4$ supersymmetric $\mathrm{AdS}_{7}$ in the limit $r \rightarrow \infty$ with

$$
U \sim W \sim 2 g r, \quad \phi \sim \theta \sim 0 .
$$

It should be noted that in this limit, the main contribution to the solution is obtained from the scalar. The contribution from the three-form field strength is highly suppressed as can be seen from its components in the flat basis given in (60). In the limit $r \rightarrow 0$, the solution is singular similar to the solution studied in [12].

For $S O(4,1)$ gauge group with $\rho=-1$, there is no $\mathrm{AdS}_{7}$ asymptotic since this gauge group does not admit a supersymmetric $\mathrm{AdS}_{7}$ vacuum. In this case, the solution is the $S O(4)$ symmetric domain wall studied in [26] with a dyonic profile of the three-form flux.

For $\operatorname{CSO}(4,0,1)$ gauge group with $\rho=0$, the BPS equations (66)-(71), with $\tau=0$, become

$$
\begin{gathered}
U^{\prime}=W^{\prime}=\frac{1}{10} g e^{V-2 \phi} \sec 2 \theta, \\
\phi^{\prime}=\frac{1}{20} g e^{V-2 \phi} \sec 2 \theta, \\
\theta^{\prime}=k=0 \\
l=-\frac{1}{4} g e^{3 W-6 \phi} \tan 2 \theta
\end{gathered}
$$

together with the constraint

$$
\kappa=-\frac{1}{2} g e^{W-2 \phi} \tan 2 \theta .
$$

Equation (92) implies that $\theta$ is constant. Note that for $\theta=0$, these equations reduce to those of the $M k w_{3} \times \mathbb{R}^{3}$ sliced domain wall.

In the present case, the constraint (94) implies that $\theta$ cannot be zero since $\kappa \neq 0$. Furthermore, a nonvanishing $\theta$ gives a nontrivial three-form flux according to (93) to support the $S^{3}$ part. For constant $\theta \neq 0$, we can find the following solution, after choosing $V=0$ gauge choice:

$$
\begin{gathered}
U=W=2 \phi, \quad k=0, \\
l=-\frac{1}{4} g \tan 2 \theta, \\
e^{2 \phi}=\frac{1}{10} g r \sec 2 \theta+2 C
\end{gathered}
$$

with an integration constant $C$. The constant $\theta$ is given by

$$
\theta=-\frac{1}{2} \tan ^{-1} \frac{2 \kappa}{g} .
$$


As in the $S O(4,1)$ gauge group, it can be verified that for a given constant $\theta$, this solution is the $S O(4)$ symmetric domain wall of $\operatorname{CSO}(4,0,1)$ gauge group given in [26] with a magnetic profile of a constant three-form flux.

\section{3. $\mathrm{AdS}_{3} \times S^{3}$-sliced domain walls}

We now consider more complicated solutions with an $\mathrm{AdS}_{3} \times S^{3}$ slice. As in [12], we begin with a simpler solution with a single warp factor $U=W$. From the BPS equations (66)-(71), imposing $U^{\prime}=W^{\prime}$ gives

$$
\theta=0, \quad k=l, \quad \tau=\kappa .
$$

Setting $\theta=0$, we find that the BPS equations become

$$
\begin{gathered}
U^{\prime}=\frac{g}{40} e^{V-2 \phi}\left(4+\rho e^{10 \phi}\right), \\
\phi^{\prime}=\frac{g}{20} e^{V-2 \phi}\left(1-\rho e^{10 \phi}\right), \\
k=\frac{1}{2} e^{2 U-4 \phi} \tau .
\end{gathered}
$$

By choosing $V=-3 \phi$, we obtain the following solution:

$$
\begin{gathered}
U=2 \phi-\frac{1}{4} \ln \left(1-\rho e^{10 \phi}\right), \\
e^{5 \phi}=\frac{1}{\sqrt{\rho}} \tanh \left[\frac{\sqrt{\rho}}{4}(g r+C)\right], \\
k=\frac{1}{2} \tau \cosh \left[\frac{\sqrt{\rho}}{4}(g r+C)\right]
\end{gathered}
$$

with an integration constant $C$. This solution is the $S O(4)$ symmetric domain wall coupled to a dyonic profile of the three-form flux.

For $S O(5)$ gauge group, the solution is locally asymptotic to the supersymmetric $\mathrm{AdS}_{7}$ dual to $N=(2,0) \mathrm{SCFT}$ in six dimensions. This solution is then expected to describe a surface defect, corresponding to the $\mathrm{AdS}_{3}$ part, within the six-dimensional $N=(2,0)$ SCFT. Similarly, according to the DW/QFT correspondence, the usual $M k w_{6}$-sliced domain wall without the three-form flux is dual to an $N=(2,0)$ nonconformal field theory in six dimensions. We then interpret the solutions for $S O(4,1)$ and $\operatorname{CSO}(4,0,1)$ gauge groups as describing a surface defect within a nonconformal $N=(2,0)$ field theory in six dimensions.

We now consider more general solutions with the $\mathrm{AdS}_{3} \times S^{3}$ slice. We will find the solutions for the cases of $\rho= \pm 1$ and $\rho=0$ separately. With the same gauge choice given in (82), the BPS equations (66)-(71) for $\rho \neq 0$ are solved by

$$
\begin{gathered}
U=2 \phi-\ln (\sin 2 \theta), \\
W=2 \phi-\ln (\tan 2 \theta),
\end{gathered}
$$

$$
e^{10 \phi}=\frac{3 g C+2 g \rho-4 \tau \rho+4(\tau \rho-g C) \cos 4 \theta+g C \cos 8 \theta}{g(\cos 4 \theta+1)},
$$

$$
\begin{gathered}
k=\frac{1}{8}\left(4 \tau \csc ^{2} 2 \theta-g \csc ^{4} 2 \theta-4 g \rho C\right) \tan ^{2} 2 \theta, \\
l=\frac{1}{8}\left(g \csc ^{2} 2 \theta-2 g \cot ^{2} 2 \theta-4 \tau+4 g \rho C \sin ^{2} 2 \theta\right),
\end{gathered}
$$

$$
\theta=\arctan \left(e^{-2 g \rho r}\right)
$$

together with the following relation obtained from the constraint (72):

$$
\kappa=-\frac{g}{2}+\tau \text {. }
$$

As in the previous case, for $S O(5)$ gauge group, the solution is locally asymptotically $\mathrm{AdS}_{7}$ given in (89) as $r \rightarrow \infty$. For $S O(4,1)$ gauge group, the solution is a charged domain wall with a nonvanishing three-form flux. In general, these solutions describe, respectively, holographic RG flows from an $N=(2,0)$ SCFT and $N=(2,0)$ nonconformal field theory to a singularity at $r=0$ except for a special case with $\tau=g(\rho C+1) / 4$. This is very similar to the solutions of pure $N=2$ gauged supergravity studied in [12].

For the particular value of $\tau=g(\rho C+1) / 4$, the scalar potential is constant as $r \rightarrow 0$, and the solution turns out to be described by a locally $\mathrm{AdS}_{3} \times T^{4}$ geometry with the following leading profile:

$$
\begin{array}{rlrlrl}
e^{2 U} & \sim(\rho-4 C)^{\frac{2}{5}}, & e^{2 W} \sim 0, & & \phi \sim \frac{1}{10} \ln (\rho-4 C), \\
\theta & \sim \frac{\pi}{4}, \quad k \sim \frac{g}{8}(4 \rho C-1), & l & \sim 0 .
\end{array}
$$

To obtain real solutions, we choose the integration constant $C<\frac{1}{4}$ and $C<-\frac{1}{4}$ for $S O(5)$ and $S O(4,1)$ gauge groups, respectively.

For $\operatorname{CSO}(4,0,1)$ gauge group with $\rho=0$, we find the following solution, after setting $V=0$,

$$
\begin{gathered}
U=W=2 \phi, \\
k=\frac{1}{2} \tau, \\
l=\frac{1}{4}(2 \tau-g \sin 2 \theta) \sec 2 \theta,
\end{gathered}
$$




$$
e^{2 \phi}=\frac{1}{10} r(g \sec 2 \theta-2 \tau \tan 2 \theta)+2 C,
$$

where the constant $\kappa$ is given by

$$
\kappa=\tau \sec 2 \theta-\frac{1}{2} g \tan 2 \theta
$$

Note also that, in this case, $\theta$ is constant since the corresponding BPS equation gives $\theta^{\prime}=0$ as can be seen from Eq. (69).

\section{Coupling to $\mathrm{SO}(3)$ gauge fields}

In this section, we extend the analysis by coupling the previously obtained solutions to $S O(3)$ vectors describing a Hopf fibration of the three-sphere. With the projector
$\left(\Gamma_{5}\right)^{a}{ }_{b} \epsilon_{0}^{b}=\epsilon_{0}^{a}$ and the identity $\Gamma_{1} \cdots \Gamma_{5}=\mathbf{I}_{4}$, we turn on the gauge fields corresponding to the anti-self-dual $S O(3) \subset S O(4)$. The ansatz for these gauge fields is chosen to be

$$
\begin{aligned}
& A_{(1)}^{23}=-A_{(1)}^{14}=e^{-W(r)} \frac{\kappa}{4} p(r) e^{\hat{4}}, \\
& A_{(1)}^{31}=-A_{(1)}^{24}=e^{-W(r)} \frac{\kappa}{4} p(r) e^{\hat{5}}, \\
& A_{(1)}^{12}=-A_{(1)}^{34}=e^{-W(r)} \frac{\kappa}{4} p(r) e^{\hat{6}} .
\end{aligned}
$$

The function $p(r)$ is the magnetic charge with the dependence on the radial coordinate. The corresponding two-form field strengths can be computed to be

$$
\begin{aligned}
& F_{(2)}^{23}=-F_{(2)}^{14}=e^{-V-W} \frac{\kappa}{4} p^{\prime} e^{\hat{3}} \wedge e^{\hat{4}}+e^{-2 W} \frac{\kappa^{2}}{8} p(2-g p) e^{\hat{5}} \wedge e^{\hat{6}}, \\
& F_{(2)}^{31}=-F_{(2)}^{24}=e^{-V-W} \frac{\kappa}{4} p^{\prime} e^{\hat{3}} \wedge e^{\hat{5}}+e^{-2 W} \frac{\kappa^{2}}{8} p(2-g p) e^{\hat{6}} \wedge e^{\hat{4}}, \\
& F_{(2)}^{12}=-F_{(2)}^{34}=e^{-V-W} \frac{\kappa}{4} p^{\prime} e^{\hat{3}} \wedge e^{\hat{6}}+e^{-2 W} \frac{\kappa^{2}}{8} p(2-g p) e^{\hat{4}} \wedge e^{\hat{5}} .
\end{aligned}
$$

For gaugings in the $\mathbf{1 5}$ representation, there are no massive two-form fields due to the vanishing $Z^{M N, P}$. The modified twoform field strengths $\mathcal{H}_{\mu \nu}^{(2) M N}$ are simply given by the $S O(3)$ gauge field strengths $F_{\mu \nu}^{M N}$.

To preserve some amount of supersymmetry, we need to impose additional projectors on the constant spinors $\epsilon_{0}^{a}$ as follows:

$$
\gamma_{\hat{4} \hat{5}} \epsilon_{0}^{a}=-\left(\Gamma_{12}\right)_{b}^{a} \epsilon_{0}^{b}, \quad \gamma_{\hat{5} \hat{b}} \epsilon_{0}^{a}=-\left(\Gamma_{23}\right)^{a}{ }_{b} \epsilon_{0}^{b}, \quad \gamma_{\hat{b} \hat{4}} \epsilon_{0}^{a}=-\left(\Gamma_{31}\right)_{b}^{a} \epsilon_{0}^{b} .
$$

It should be noted that the last projector is not independent of the first two. Therefore, together with the projectors given in (65), there are four independent projectors on $\epsilon_{0}^{a}$, and the residual supersymmetry consists of two supercharges.

With all these, the resulting BPS equations for the $\mathrm{AdS}_{3} \times S^{3}$-sliced domain wall are given by

$$
\begin{gathered}
U^{\prime}=\frac{e^{V-2(W+\phi)}}{80 \cos 2 \theta}\left[e^{2 W}\left(g\left(4+\rho e^{10 \phi}\right)(3 \cos 4 \theta-1)+32 e^{2 \phi-U} \tau \sin 2 \theta\right)\right. \\
\left.+12 e^{4 \phi}\left(\kappa^{2} p(g p-2)(\cos 4 \theta-3)+2 e^{W-2 \phi} \kappa(g p-1) \sin 4 \theta\right)\right], \\
W^{\prime}=\frac{e^{V-2(W+\phi)}}{40 \cos 2 \theta}\left[e^{2 W}\left(g\left(4+\rho e^{10 \phi}\right)(2-\cos 4 \theta)+24 e^{2 \phi-U} \tau \sin 2 \theta\right)\right. \\
\left.+4 e^{4 \phi}\left(\kappa^{2} p(g p-2)(\cos 4 \theta-8)-2 e^{W-2 \phi} \kappa(g p-1) \sin 4 \theta\right)\right], \\
\phi^{\prime}=\frac{e^{V-2(W+\phi)}}{80 \cos 2 \theta}\left[e^{2 W}\left(g\left(6 \cos 4 \theta-2-\rho e^{10 \phi}(\cos 4 \theta+3)\right)+16 e^{2 \phi-U} \tau \sin 2 \theta\right)\right. \\
\left.+6 e^{4 \phi}\left(\kappa^{2} p(g p-2)(3-\cos 4 \theta)+2 e^{W-2 \phi} \kappa(g p-1) \sin 4 \theta\right)\right], \\
\theta^{\prime}=\frac{e^{V-2(W+\phi)}}{16}\left[24 e^{W+2 \phi}\left(e^{W-U} \tau+\kappa(g p-1) \cos 2 \theta\right)\right. \\
\left.-\left(g e^{2 W}\left(12+\rho e^{10 \phi}\right)-12 e^{4 \phi} \kappa^{2} p(g p-2)\right) \sin 2 \theta\right],
\end{gathered}
$$




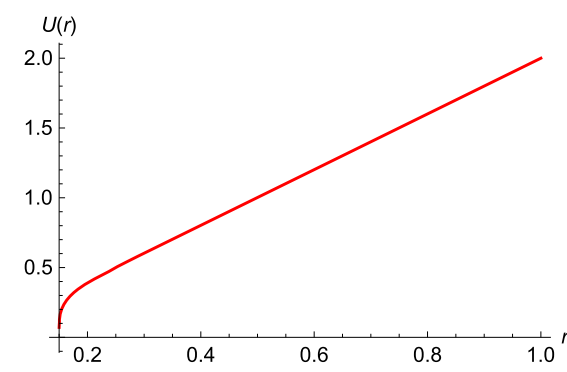

(a) $U$ solution

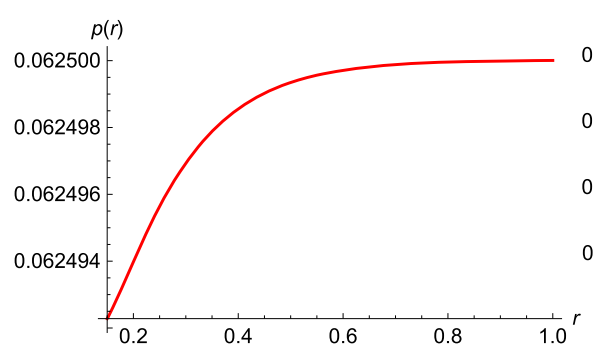

(d) $p$ solution

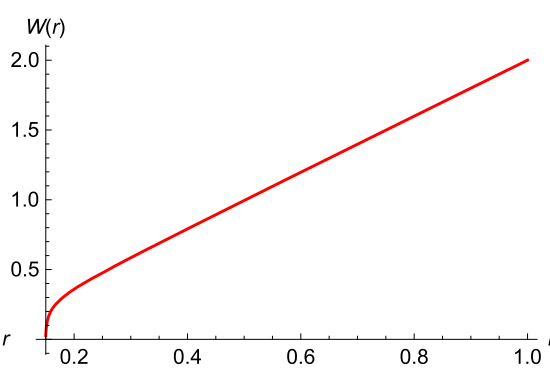

(b) $W$ solution

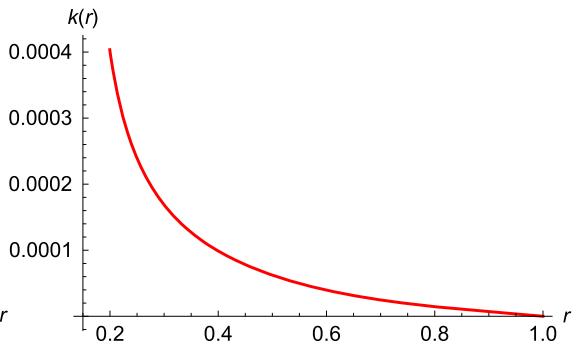

(e) $k$ solution

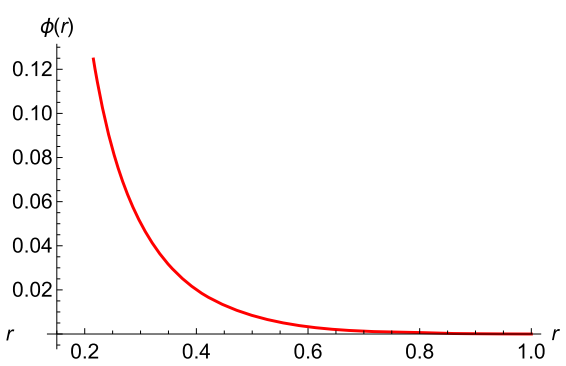

(c) $\phi$ solution

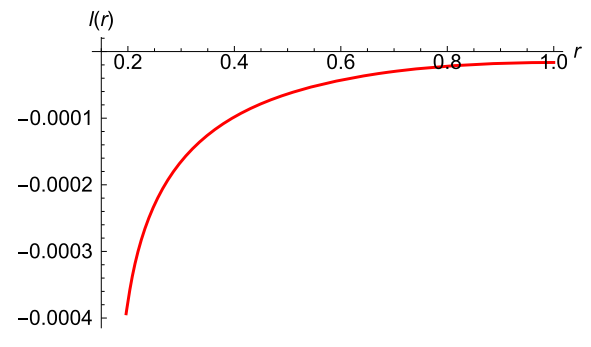

(f) $l$ solution

FIG. 1. A BPS flow from a locally $\mathrm{AdS}_{7}$ geometry at $r \rightarrow \infty$ to the singularity at $r=0$ for the $M k w_{3} \times S^{3}$-sliced domain wall with $\tau=0$.

$$
\begin{gathered}
k=\frac{1}{8} e^{3 U-4 \phi}\left(4 e^{-U} \tau-g \rho e^{8 \phi} \sin 2 \theta\right) \\
l=\frac{1}{8} e^{3 W-6 \phi}\left[g\left(4+\rho e^{10 \phi}\right) \tan 2 \theta-8 e^{2 \phi-U} \tau \sec 2 \theta\right. \\
\left.-12 e^{4 \phi-2 W}\left(\kappa^{2} p(g p-2) \tan 2 \theta+e^{W-2 \phi} \kappa(g p-1)\right)\right] \\
p^{\prime}=\frac{e^{V-W-4 \phi}}{2 \kappa}\left[2 e^{W+2 \phi}\left(e^{W-U} \tau+\kappa(g p-1) \cos 2 \theta\right)\right. \\
\left.-\left(g e^{2 W}-e^{4 \phi} \kappa^{2} p(g p-2)\right) \sin 2 \theta\right] .
\end{gathered}
$$

In contrast to the previous case, it can also be verified that these equations satisfy the second-order field equations without imposing any constraint. By setting $\tau=0$, we can obtain the BPS equations for a $M k w_{3} \times S^{3}$-sliced domain wall. For $p(r)=0$, we obtain the BPS equations (66)-(71) for charged domain walls without gauge fields. In this case, Eq. (132) becomes the algebraic constraint (72).

The BPS equations in this case are much more complicated, and we are not able to find analytic flow solutions. We then look for numerical solutions with some appropriate boundary conditions. We first consider the solutions in $S O(5)$ gauge group with an $\mathrm{AdS}_{7}$ asymptotic at large $r$. With $\rho=1$, we find that the following locally $\mathrm{AdS}_{7}$ configuration solves the BPS equations at the leading order as $r \rightarrow \infty$ :

$$
U \sim W \sim \frac{r}{L}, \quad \phi \sim \theta \sim 0, \quad p \sim \frac{1}{g}\left(1-\frac{\tau}{\kappa}\right)
$$

with $L=\frac{8}{g}$. With this boundary condition and $V=0$ gauge choice, we find some examples of the BPS flows from this locally $\mathrm{AdS}_{7}$ geometry as $r \rightarrow \infty$ to the singularity at $r=0$ as shown in Figs. 1 and 2 for $g=16$ and $\kappa=2$. It should be noted that we have not imposed the boundary conditions on $k$ and $l$ since the corresponding BPS equations are algebraic. This is rather different from the solutions in [12] in which the BPS equations for $k$ and $l$ are differential.

From the numerical solution in Fig. 2, the solutions for $k$ and $l$ appear to be diverging as $k \sim e^{2 U}$ and $l \sim e^{2 W}$ for $r \rightarrow \infty$. However, the contribution from the three-form flux is sufficiently suppressed for $r \rightarrow \infty$ since the terms involving $\mathcal{H}_{5}^{(3)}$ in the BPS equations behave as $k e^{-3 U}+l e^{-3 W}$.

For $\operatorname{SO}(4,1)$ and $C S O(4,0,1)$ gauge groups, there is no locally asymptotic $\mathrm{AdS}_{7}$ configuration. However, we can look for solutions of the BPS equations (126)-(132) in the form of a flow from the charged domain wall without vector fields given previously to the singularity at $r=0$. 


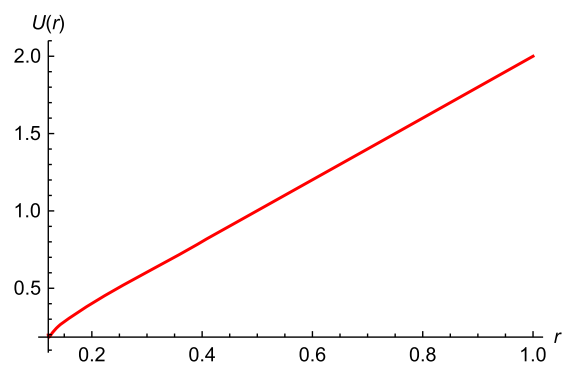

(a) $U$ solution

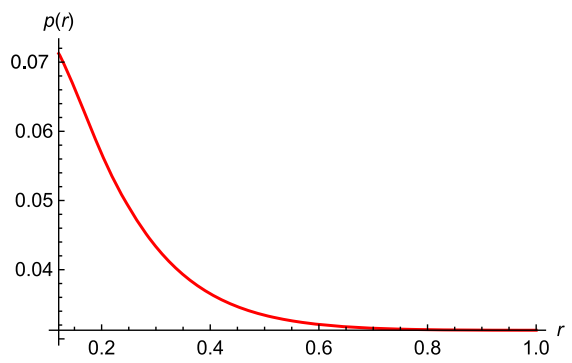

(d) $p$ solution

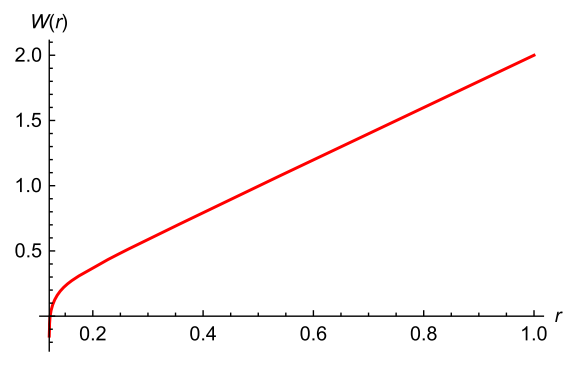

(b) $W$ solution

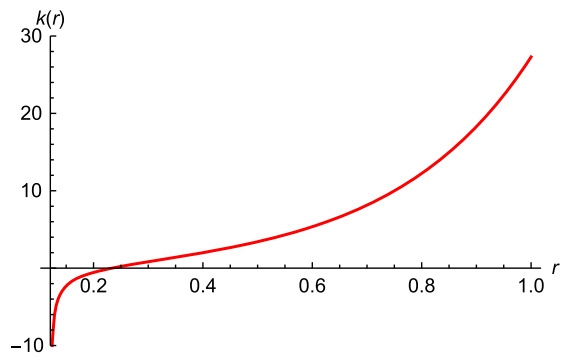

(e) $k$ solution

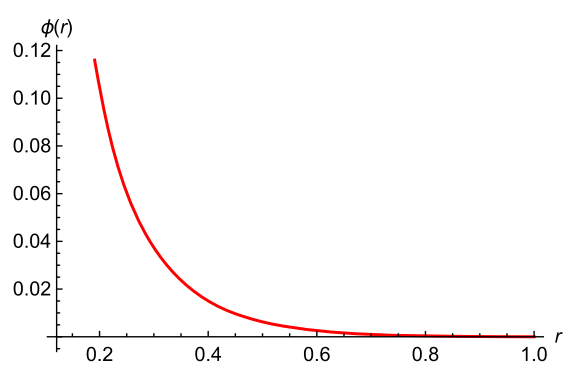

(c) $\phi$ solution

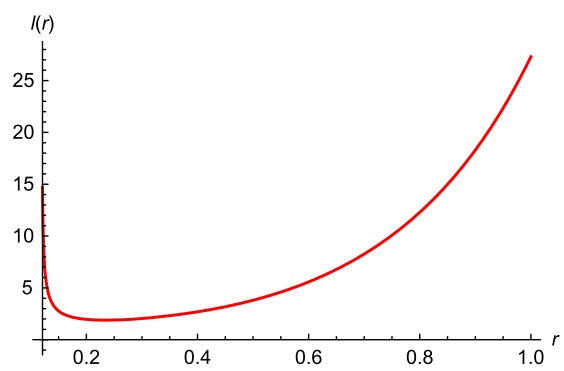

(f) $l$ solution

FIG. 2. A BPS flow from a locally $\mathrm{AdS}_{7}$ geometry at $r \rightarrow \infty$ to the singularity at $r=0$ for the $\operatorname{AdS}_{3} \times S^{3}$-sliced domain wall with $\tau=1$.

We first choose the gauge choice $V=-3 \phi$ and consider the following behavior at the leading order when $g r \rightarrow C$, for a constant $C$,

$U \sim W \sim \frac{2}{5} \ln (g r-C), \quad \phi \sim \frac{1}{5} \ln (g r-C)$,

$\theta \sim p \sim 0, \quad$ and $\quad k \sim l \sim \frac{\tau}{2}$

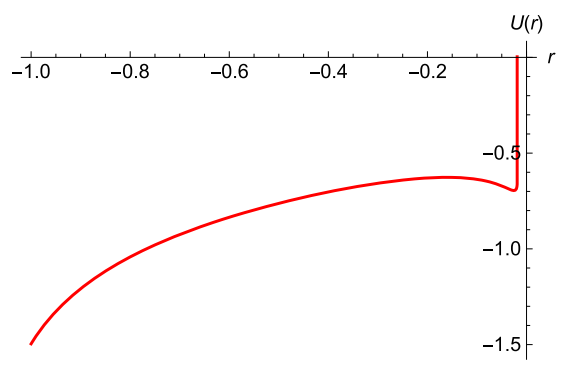

(a) $U$ solution

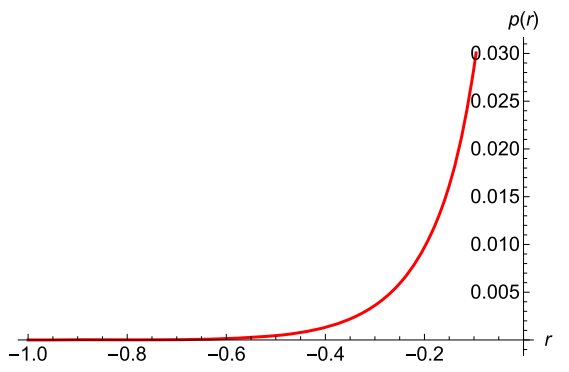

(d) $p$ solution with $\kappa=\tau$. It can be verified that this configuration solves the BPS equations (66)-(72) in the limit $g r \rightarrow C$. Since this configuration also appears in $S O(5)$ gauge group, we will consider the solutions for $S O(5)$ gauge group as well.

Examples of the BPS flows from the charged domain wall in (134) as $g r \rightarrow C$ to the singularity at $r=0$ in $S O(5), S O(4,1)$, and $C S O(4,0,1)$ gauge groups are shown in Figs. 3-5, respectively. In these solutions, we

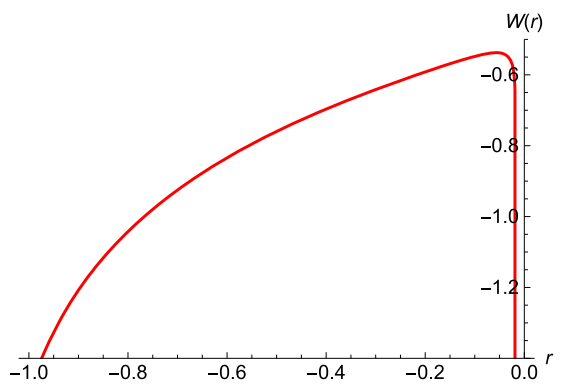

(b) $W$ solution

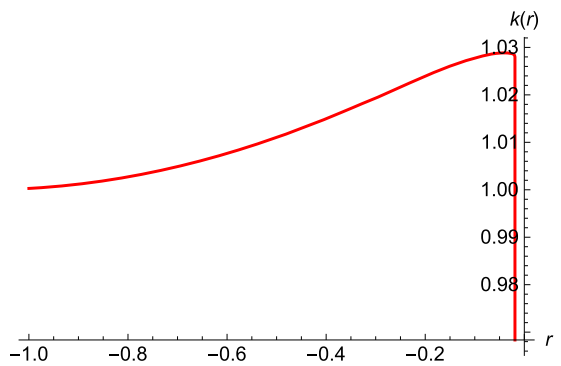

(e) $k$ solution

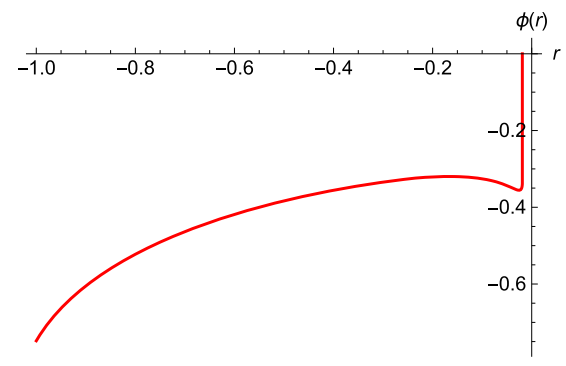

(c) $\phi$ solution

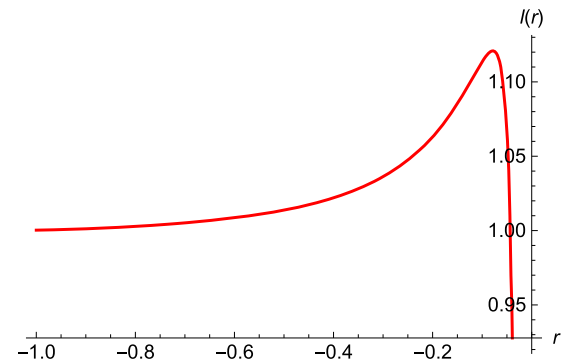

(f) $l$ solution

FIG. 3. A BPS flow from a charged domain wall at $r=-1$ to the singularity at $r=0$ in $S O(5)$ gauge group. 


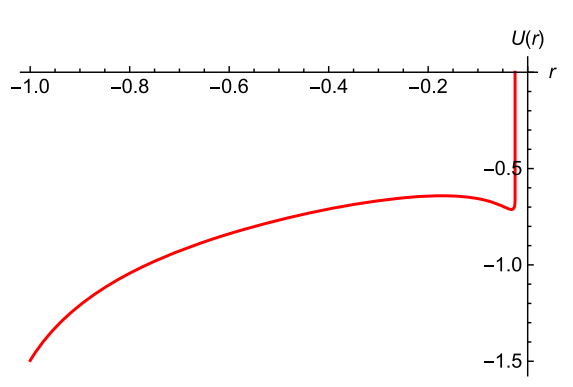

(a) $U$ solution

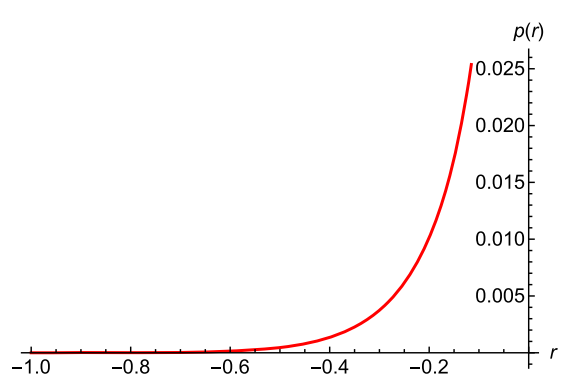

(d) $p$ solution

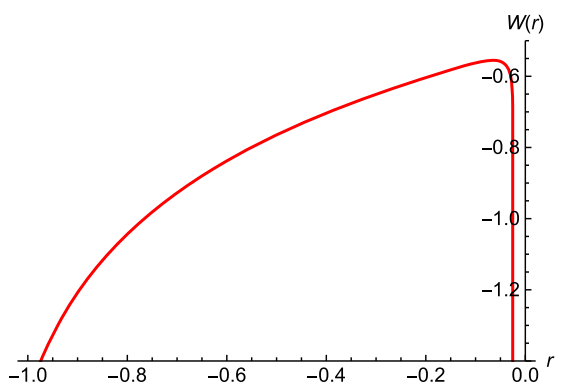

(b) $W$ solution

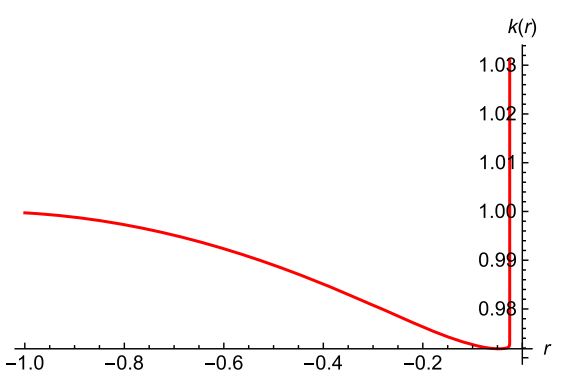

(e) $k$ solution

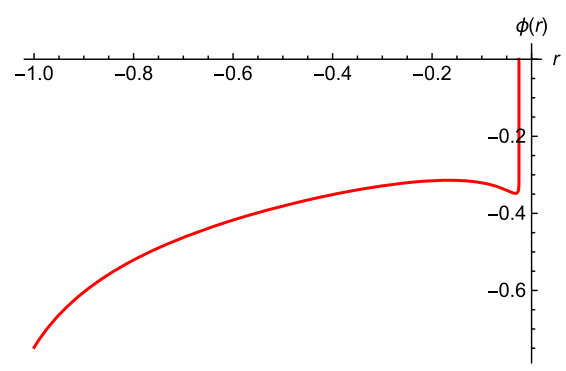

(c) $\phi$ solution

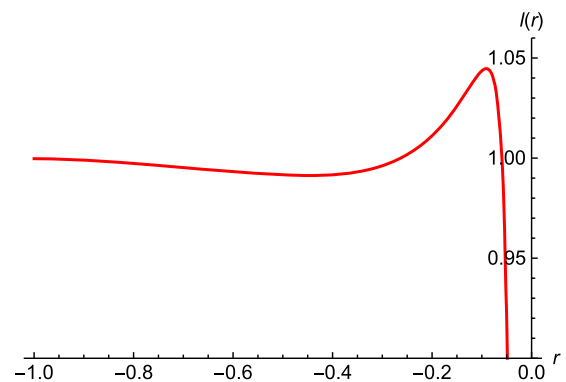

(f) $l$ solution

FIG. 4. A BPS flow from a charged domain wall at $r=-1$ to the singularity at $r=0$ in $S O(4,1)$ gauge group.

have chosen the following numerical values $g=1$, $\kappa=\tau=2$, and $C=-1$. These solutions should describe surface defects within $N=(2,0)$ nonconformal field theories in six dimensions. For the solution in Fig. 5, $k$ is constant since, for $\rho=0$, the BPS equations (126) and (128) give constant $U-2 \phi$.
For $S O(5)$ gauge group, it is also possible to find flow solutions between the asymptotically locally $\mathrm{AdS}_{7}$ geometry and the charged domain wall configuration with an intermediate singularity in the presence of nonvanishing vector fields at $r=0$. With the gauge choice $V=-3 \phi$ and $g=1, \kappa=\tau=2$ and $C=-1$, an example of these

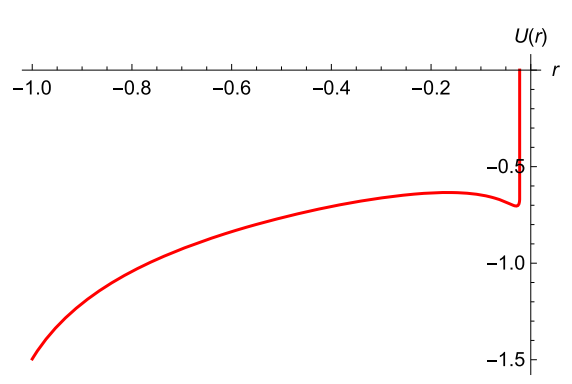

(a) $U$ solution

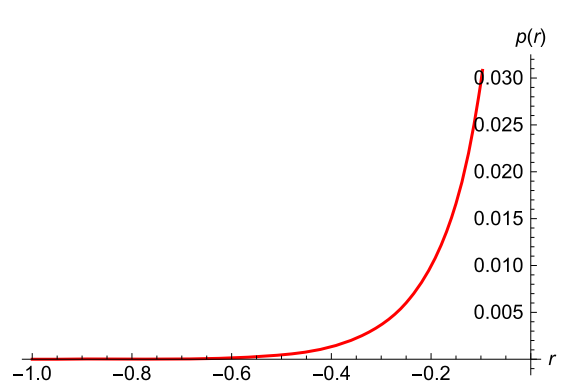

(d) $p$ solution

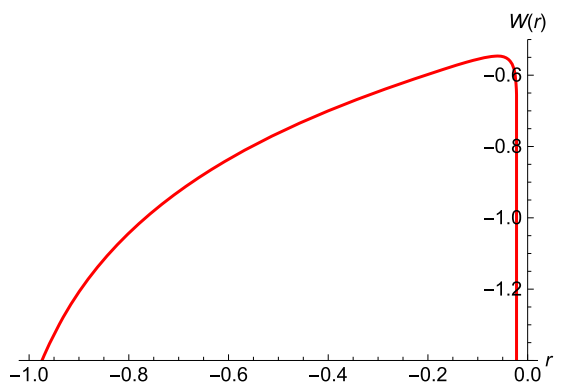

(b) $W$ solution

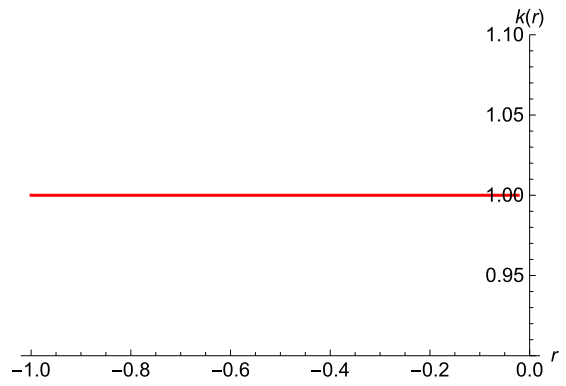

(e) $k$ solution

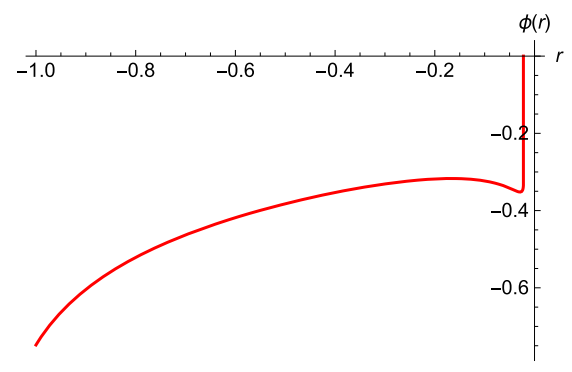

(c) $\phi$ solution

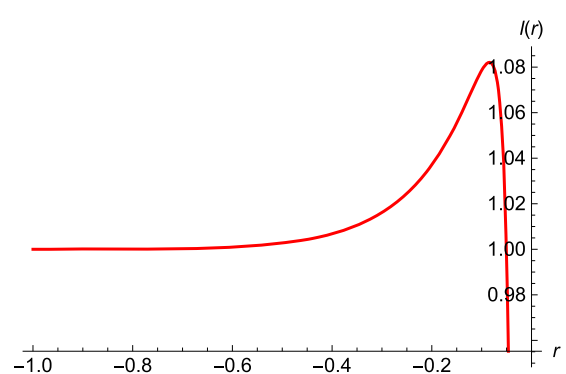

(f) $l$ solution

FIG. 5. A BPS flow from a charged domain wall at $r=-1$ to the singularity at $r=0$ in $\operatorname{CSO}(4,0,1)$ gauge group. 


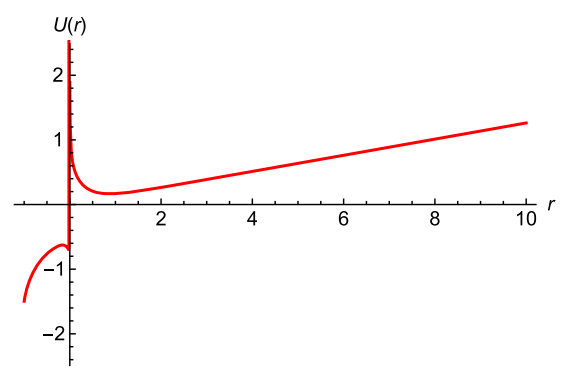

(a) $U$ solution

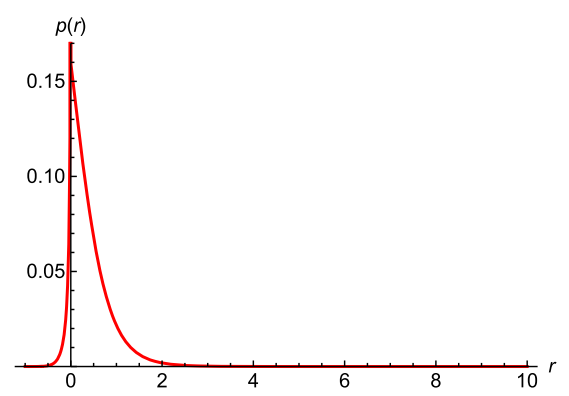

(d) $p$ solution

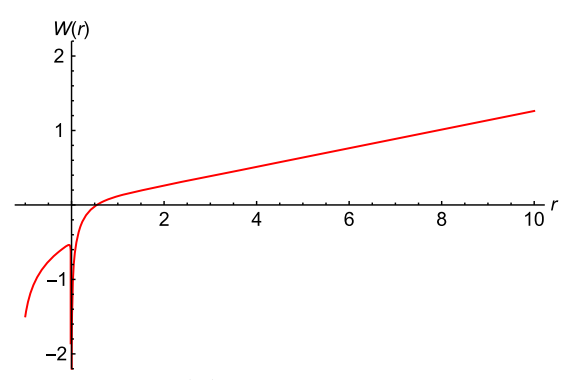

(b) $W$ solution

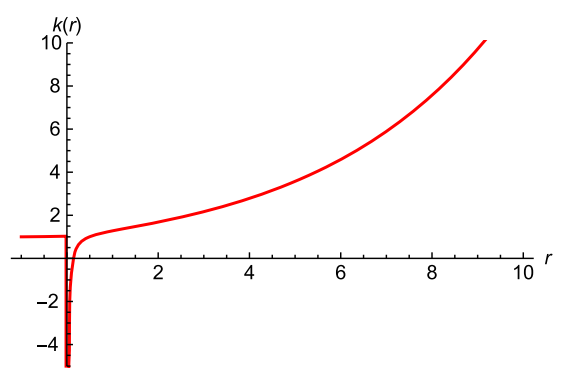

(e) $k$ solution

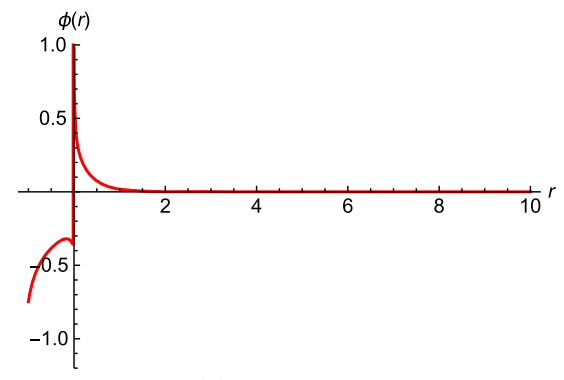

(c) $\phi$ solution

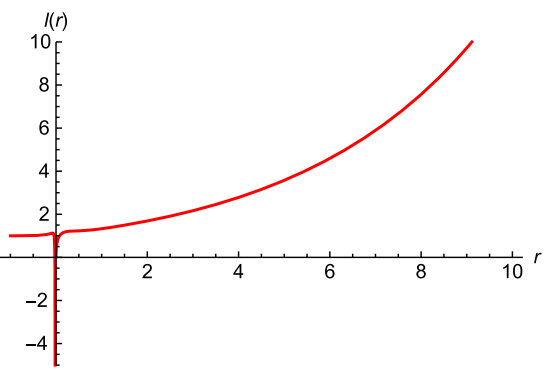

(f) $l$ solution

FIG. 6. A BPS flow between a charged domain wall at $r=-1$ and an asymptotically locally AdS geometry as $r \rightarrow \infty$ with an intermediate singularity at $r=0$ in $S O(5)$ gauge group.

solutions is shown in Fig. 6. In this solution, it is clearly seen that the vector fields vanish at both ends of the flow with a singularity at $r=0$.

\section{B. $S O(3)$ symmetric charged domain walls}

In this section, we consider charged domain walls preserving $S O(3)$ residual symmetry. There are three singlet scalars corresponding to the following noncompact generators:

$$
\begin{aligned}
& \hat{Y}_{1}=2 e_{1,1}+2 e_{2,2}+2 e_{3,3}-3 e_{4,4}-3 e_{5,5}, \\
& \hat{Y}_{2}=e_{4,5}+e_{5,4}, \\
& \hat{Y}_{3}=e_{4,4}-e_{5,5} .
\end{aligned}
$$

There are many possible gauge groups with an $S O(3)$ subgroup. To accommodate all of these gauge groups in a single framework, we use the embedding tensor of the form

$$
Y_{M N}=\operatorname{diag}(+1,+1,+1, \sigma, \rho) .
$$

For different values of $\rho, \sigma=0, \pm 1$, this embedding tensor gives rise to the following gauge groups: $S O(5)(\rho=$ $\sigma=1), S O(4,1)(-\rho=\sigma=1), S O(3,2)(\rho=\sigma=-1)$, $\operatorname{CSO}(4,0,1) \quad(\rho=0, \quad \sigma=1), \quad \operatorname{CSO}(3,1,1) \quad(\rho=0$, $\sigma=-1)$, and $\operatorname{CSO}(3,0,2)(\rho=\sigma=0)$. The unbroken $S O(3)$ symmetry is generated by $X_{M N}, M, N=1,2,3$, generators.

With the $S L(5) / S O(5)$ coset representative of the form

$$
\mathcal{V}=e^{\phi_{1} \hat{Y}_{1}+\phi_{2} \hat{Y}_{2}+\phi_{3} \hat{Y}_{3}}
$$

the scalar potential reads

$$
\begin{aligned}
\mathbf{V}= & -\frac{g^{2}}{64}\left[3 e^{-8 \phi_{1}}+6 e^{2 \phi_{1}}\left[(\rho+\sigma) \cosh 2 \phi_{2} \cosh 2 \phi_{3}+(\rho-\sigma) \sinh 2 \phi_{3}\right]\right. \\
& +\frac{1}{4} e^{12 \phi_{1}}\left[\rho^{2}+10 \rho \sigma+\sigma^{2}-\left(3 \rho^{2}-2 \rho \sigma+3 \sigma^{2}\right) \cosh 4 \phi_{3}\right. \\
& \left.\left.-(\rho+\sigma)^{2} \cosh 4 \phi_{2}\left(1+\cosh 4 \phi_{3}\right)-4\left(\rho^{2}-\sigma^{2}\right) \cosh 2 \phi_{2} \sinh 4 \phi_{3}\right]\right] .
\end{aligned}
$$

For $S O(5)$ gauge group, this potential admits a supersymmetric $\mathrm{AdS}_{7}$ vacuum given in (58) at $\phi_{1}=\phi_{2}=\phi_{3}=0$ and a nonsupersymmetric $\mathrm{AdS}_{7}$ given in (59) at $\phi_{1}=\frac{1}{20} \ln 2, \phi_{2}= \pm \frac{1}{4} \ln 2$, and $\phi_{3}=0$. 
We now repeat the same procedure as in the previous section to set up the BPS equations. The $S O(3)$ residual symmetry allows for two three-form field strengths, $\mathcal{H}_{\mu \nu \rho M}^{(3)}$ with $M=4$, 5. We will choose the following ansatz:

$$
\begin{array}{ll}
\mathcal{H}_{\hat{m} \hat{n} \hat{p} 4}^{(3)}=k_{4}(r) e^{-3 U(r)} \varepsilon_{\hat{m} \hat{n} \hat{p}}, & \mathcal{H}_{\hat{i} \hat{j} \hat{k} 4}^{(3)}=l_{4}(r) e^{-3 W(r)} \varepsilon_{\hat{i} \hat{j} \hat{k}}, \\
\mathcal{H}_{\hat{m} \hat{n} \hat{p} 5}^{(3)}=k_{5}(r) e^{-3 U(r)} \varepsilon_{\hat{m} \hat{n} \hat{p}}, & \mathcal{H}_{\hat{i} \hat{j} \hat{k} 5}^{(3)}=l_{5}(r) e^{-3 W(r)} \varepsilon_{\hat{i} \hat{j} \hat{k}} .
\end{array}
$$

With $\mathcal{H}_{\mu \nu \rho 4}^{(3)}$ nonvanishing, the $S O(5)$ gamma matrix $\Gamma_{4}$ will appear in the BPS conditions. To avoid an additional projector, which will break more supersymmetry, we impose the following condition:

$$
k_{4}(r)=\tanh \phi_{2} k_{5}(r) \quad \text { and } \quad l_{4}(r)=\tanh \phi_{2} l_{5}(r) .
$$

This simply makes the coefficient of $\Gamma_{4}$ vanish. It would also be interesting to consider a more general projector.

With the projection conditions in (65), we can find a consistent set of BPS equations for

$$
\theta=0 \quad \text { and } \quad \tau=e^{U-W} \kappa .
$$

The latter forbids the possibility of setting either $\tau=0$ or $\kappa=0$ without ending up with $\kappa=\tau=0$. Therefore, the solutions in this case can only be $\mathrm{AdS}_{3} \times S^{3}$-sliced domain walls.

The resulting BPS equations take the form

$$
\begin{gathered}
U^{\prime}=\frac{g}{40} e^{V+6 \phi_{1}}\left(3 e^{-10 \phi_{1}}+(\rho+\sigma) \cosh 2 \phi_{2} \cosh 2 \phi_{3}+(\rho-\sigma) \sinh 2 \phi_{3}\right), \\
W^{\prime}=\frac{g}{40} e^{V+6 \phi_{1}}\left(3 e^{-10 \phi_{1}}+(\rho+\sigma) \cosh 2 \phi_{2} \cosh 2 \phi_{3}+(\rho-\sigma) \sinh 2 \phi_{3}\right), \\
\phi_{1}^{\prime}=\frac{g}{40} e^{V+6 \phi_{1}}\left(2 e^{-10 \phi_{1}}-(\rho+\sigma) \cosh 2 \phi_{2} \cosh 2 \phi_{3}-(\rho-\sigma) \sinh 2 \phi_{3}\right), \\
\phi_{2}^{\prime}=-\frac{g}{8} e^{V+6 \phi_{1}}(\rho+\sigma) \sinh 2 \phi_{2} \operatorname{sech} 2 \phi_{3}, \\
\phi_{3}^{\prime}=-\frac{g}{8} e^{V+6 \phi_{1}}\left((\rho+\sigma) \cosh 2 \phi_{2} \sinh 2 \phi_{3}+(\rho-\sigma) \cosh 2 \phi_{3}\right), \\
k_{5}=\frac{1}{2} e^{3 U-W-3 \phi_{1}-\phi_{3}} \cosh \phi_{2} \kappa, \\
l_{5}=\frac{1}{2} e^{2 W-3 \phi_{1}-\phi_{3}} \cosh \phi_{2} \kappa .
\end{gathered}
$$

However, the compatibility between these BPS equations and the corresponding field equations requires either $\phi_{2}=$ 0 or $\phi_{3}=0$. It should be noted that setting $\phi_{3}=0$ is consistent with Eq. (147), namely $\phi_{3}^{\prime}=0$, only for $\sigma=\rho$, so solutions with vanishing $\phi_{3}$ can only be obtained in $S O(5), S O(3,2)$, and $\operatorname{CSO}(3,0,2)$ gauge groups. To find explicit solutions, we separately consider various possible values of $\rho$ and $\sigma$.

\section{Charged domain walls in $\mathrm{CSO}(3,0,2)$ gauge group}

For the simplest $\operatorname{CSO}(3,0,2)$ gauge group corresponding to $\rho=\sigma=0$, we find $\phi_{2}^{\prime}=\phi_{3}^{\prime}=0$, so we can consistently set $\phi_{3}=0$ and $\phi_{2}=0$. With $\phi_{2}=0$,
Eq. (141) gives $k_{4}=l_{4}=0$. Choosing $V=0$ gauge choice, we find the following charged domain wall solution:

$$
\begin{gathered}
U=W=\frac{3}{8} \ln \left[\frac{g r}{5}+C\right], \\
\phi_{1}=\frac{1}{4} \ln \left[\frac{g r}{5}+C\right], \\
\text { and } \quad k_{5}=l_{5}=\frac{1}{2} \tau
\end{gathered}
$$

with an integration constant $C$. 


\section{Charged domain walls in $\mathrm{CSO}(4,0,1)$ and $\operatorname{CSO}(3,1,1)$ gauge groups}

In this case, we have $\rho=0$ and $\sigma= \pm 1$ corresponding to $\operatorname{CSO}(4,0,1)(\sigma=+1)$ and $\operatorname{CSO}(3,1,1)(\sigma=-1)$ gauge groups. Choosing $V=-6 \phi_{1}$ gauge choice, we can find a charged domain wall solution, with $\phi_{2}=0$,

$$
\begin{gathered}
\phi_{3}=\frac{1}{2} \ln \left[\frac{g \sigma r}{4}+C_{1}\right], \\
\phi_{1}=-\frac{1}{5} \phi_{3}+\frac{1}{10} \ln \left[C_{2}+e^{4 \phi_{3}}\right], \\
U=W=\frac{1}{5} \phi_{3}+\frac{3}{20} \ln \left[C_{2}+e^{4 \phi_{3}}\right], \\
k_{4}=l_{4}=0 \quad \text { and } \quad k_{5}=l_{5}=\frac{1}{2} \tau,
\end{gathered}
$$

where $C_{1}$ and $C_{2}$ are integration constants. For these gauge groups, it is not possible to find solutions with $\phi_{3}=0$.

\section{Charged domain walls in $S O(4,1)$ gauge group}

In this case, the gauge group is a noncompact $S O(4,1)$ with $\sigma=-\rho=1$. As in the previous case, it is not possible to set $\phi_{3}=0$, so we only consider solutions with $\phi_{2}=0$. Using the same gauge choice $V=-6 \phi_{1}$, we find the following solution:

$$
\begin{gathered}
e^{2 \phi_{3}}=\tan \left[\frac{g r}{4}+C_{1}\right], \\
\phi_{1}=-\frac{1}{5} \phi_{3}+\frac{1}{10} \ln \left[C_{2}\left(e^{4 \phi_{3}}+1\right)-1\right] \\
U=W=\frac{1}{5} \phi_{3}-\frac{1}{4} \ln \left[e^{4 \phi_{3}}+1\right]+\frac{3}{20} \ln \left[C_{2}\left(e^{4 \phi_{3}}+1\right)-1\right],
\end{gathered}
$$

$$
\begin{gathered}
k_{4}=l_{4}=0, \\
k_{5}=l_{5}=\frac{1}{2} \tau \cos \left[\frac{g r}{4}+C_{1}\right] .
\end{gathered}
$$

\section{Charged domain walls in $\mathrm{SO}(5)$ and $\mathrm{SO}(3,2)$ gauge groups}

We now look at the last possibility with $\rho=\sigma= \pm 1$ corresponding to $S O(5)$ and $S O(3,2)$ gauge groups. In this case, it is possible to set $\phi_{2}=0$ or $\phi_{3}=0$. With $\phi_{2}=0$ and $V=-6 \phi_{1}$, we find the following solution:

$$
\begin{gathered}
\phi_{3}=\frac{1}{2} \ln \left[\frac{e^{\frac{g \rho r}{2}}-C_{1}}{e^{\frac{g \rho r}{2}}+C_{1}}\right], \\
\phi_{1}=-\frac{1}{5} \phi_{3}+\frac{1}{10} \ln \left[C_{2}\left(e^{4 \phi_{3}}-1\right)+1\right],
\end{gathered}
$$

$$
U=W=\frac{1}{5} \phi_{3}-\frac{1}{4} \ln \left[e^{4 \phi_{3}}-1\right]+\frac{3}{20} \ln \left[C_{2}\left(e^{4 \phi_{3}}-1\right)+1\right]
$$

together with

$$
k_{4}=l_{4}=0 \quad \text { and } \quad k_{5}=l_{5}=\frac{\tau}{2 \sqrt{e^{4 \phi_{3}}-1}} .
$$

For $\phi_{3}=0$, we find the same solution as in (162)-(164) with $\phi_{3}$ replaced by $\phi_{2}$, but the solutions for $k_{4,5}$ and $l_{4,5}$ are now given by

$k_{4}=l_{4}=\frac{\left(e^{2 \phi_{2}}-1\right) \tau}{4 \sqrt{e^{4 \phi_{2}}-1}} \quad$ and $\quad k_{5}=l_{5}=\frac{\left(e^{2 \phi_{2}}+1\right) \tau}{4 \sqrt{e^{4 \phi_{2}}-1}}$.

Unlike the previous cases, this solution has two nonvanishing three-form fluxes.

We end this section by giving a comment on solutions with nonvanishing $S O(3)$ gauge fields. Repeating the same procedure as in the $S O(4)$ symmetric solutions leads to a set of BPS equations together with the following constraints:

$$
p^{\prime}=0 \quad \text { and } \quad p=\frac{\kappa-\tau e^{W-U}}{g \kappa} .
$$

It turns out that, in this case, the compatibility between the resulting BPS equations and the corresponding field equations requires that

$$
\tau\left(e^{W} \tau-e^{U} \kappa\right)=0 .
$$

For $\tau=0$, we can have a constant magnetic charge $p$ as required by the conditions in (167), but in this case, the three-form flux vanishes unless $e^{W} \tau=e^{U} \kappa$ as required by (142). This case corresponds to performing a topological twist along the $S^{3}$ part. Since this type of solutions is not the main aim of this paper, we will not consider them here. On the other hand, setting $e^{W} \tau=e^{U} \kappa$ does lead to nonvanishing three-form fluxes, but Eq. (167) gives vanishing gauge fields. This corresponds to the charged domain walls given above. Therefore, there does not seem to be solutions with both $S O(3)$ gauge fields and three-form fluxes nonvanishing at least for the ansatz considered here. This is very similar to the result of [13] in the matter-coupled $N=2$ gauged supergravity.

\section{C. $S O(2) \times S O(2)$ symmetric charged domain walls}

We finally consider charged domain walls with $S O(2) \times$ $S O(2)$ symmetry generated by $X_{12}$ and $X_{34}$. There are two $S O(2) \times S O(2)$ invariant scalars corresponding to the noncompact generators 
$\tilde{Y}_{1}=e_{1,1}+e_{2,2}-2 e_{5,5} \quad$ and $\quad \tilde{Y}_{2}=e_{3,3}+e_{4,4}-2 e_{5,5}$.

The $S L(5) / S O(5)$ coset representative can be written as

$$
\mathcal{V}=e^{\phi_{1} \tilde{Y}_{1}+\phi_{2} \tilde{Y}_{2}}
$$

The embedding tensor giving rise to gauge groups with an $S O(2) \times S O(2)$ subgroup is given by

$$
Y_{M N}=\operatorname{diag}(+1,+1, \sigma, \sigma, \rho)
$$

with $\rho, \sigma=0, \pm 1$. These gauge groups are $\operatorname{SO}(5)(\rho=$ $\sigma=1), S O(4,1)(-\rho=\sigma=1), S O(3,2)(\rho=-\sigma=1)$, $\operatorname{CSO}(4,0,1) \quad(\rho=0, \quad \sigma=1), \quad$ and $\quad \operatorname{CSO}(2,2,1)$ $(\rho=0, \sigma=-1)$.

Using the coset representative (170), we obtain the scalar potential

$$
\begin{aligned}
\mathbf{V}= & -\frac{1}{64} g^{2} e^{-2\left(\phi_{1}+\phi_{2}\right)}\left[8 \sigma-\rho^{2} e^{10\left(\phi_{1}+\phi_{2}\right)}\right. \\
& \left.+4 \rho\left(e^{4 \phi_{1}+6 \phi_{2}}+\sigma e^{6 \phi_{1}+4 \phi_{2}}\right)\right] .
\end{aligned}
$$

As in the previous case, a consistent set of BPS equations can be found only for $\theta=0$ and $\tau e^{W}=\kappa e^{U}$. With the three-form flux (60), which is manifestly invariant under $S O(2) \times S O(2)$, and the projectors given in (65), the resulting BPS equations read

$$
\begin{gathered}
U^{\prime}=W^{\prime}=\frac{g}{40} e^{V}\left(2 e^{-2 \phi_{1}}+\rho e^{4\left(\phi_{1}+\phi_{2}\right)}+2 \sigma e^{-2 \phi_{2}}\right), \\
\phi_{1}^{\prime}=\frac{g}{20} e^{V}\left(3 e^{-2 \phi_{1}}-\rho e^{4\left(\phi_{1}+\phi_{2}\right)}-2 \sigma e^{-2 \phi_{2}}\right), \\
\phi_{2}^{\prime}=\frac{g}{20} e^{V}\left(3 \sigma e^{-2 \phi_{2}}-\rho e^{4\left(\phi_{1}+\phi_{2}\right)}-2 e^{-2 \phi_{1}}\right), \\
k=\frac{1}{2} e^{2 U-2\left(\phi_{1}+\phi_{2}\right)} \tau, \\
l=\frac{1}{2} e^{3 W-U-2\left(\phi_{1}+\phi_{2}\right)} \tau .
\end{gathered}
$$

By choosing $V=2 \phi_{1}$, we obtain the solution

$$
\begin{gathered}
\phi_{1}=-\frac{1}{10} \ln \left[e^{C_{1}-\frac{g r}{2}}+\rho\right]-\frac{1}{5} \ln \left[e^{C_{2}-\frac{g r}{2}}+\sigma\right], \\
\phi_{2}=-\frac{3}{2} \phi_{1}-\frac{1}{4} \ln \left[e^{C_{1}-\frac{g r}{2}}+\rho\right], \\
U=W=\frac{1}{8} g r+\frac{1}{20} \ln \left[e^{C_{1}-\frac{g r}{2}}+\rho\right]+\frac{1}{10} \ln \left[e^{C_{2}-\frac{g r}{2}}+\sigma\right], \\
k=l=\frac{1}{2} \tau e^{\frac{g r}{4}} \sqrt{e^{C_{1}-\frac{g r}{2}}+\rho}
\end{gathered}
$$

with the integration constants $C_{1}$ and $C_{2}$. This solution is just the $S O(2) \times S O(2)$ symmetric domain wall found in [26] with a dyonic profile for the three-form flux. In this case, coupling to $S O(3)$ gauge fields is not possible due to the absence of any unbroken $S O(3)$ gauge symmetry.

\section{Uplifted solutions in $\mathbf{1 0}$ and $\mathbf{1 1}$ dimensions}

We now give the uplifted solutions in the case of $S O(5)$ and $\operatorname{CSO}(4,0,1)$, which can be obtained from consistent truncations of 11-dimensional supergravity on $S^{4}$ and type IIA theory on $S^{3}$, respectively. As shown in [27], other gauge groups of the form $\operatorname{CSO}(p, q, 5-p-q)$ with the embedding tensor in $\mathbf{1 5}$ representation can also be obtained from truncations of 11-dimensional supergravity on $H^{p, q} \circ T^{5-p-q}$. However, in this paper, we will not consider uplifted solutions for these gauge groups since the complete truncation ansatze have not been constructed so far. Furthermore, we will not consider uplifting solutions with nonvanishing vector fields since, in this case, the uplifted solutions are not very useful due to the lack of analytic solutions.

\section{Uplift to 11 dimensions}

We first consider uplifting the seven-dimensional solutions in $S O(5)$ gauge group to 11-dimensional supergravity. We begin with the $S O(4)$ symmetric solution with the $S L(5) / S O(5)$ scalar matrix

$$
\mathcal{M}_{M N}=\operatorname{diag}\left(e^{2 \phi}, e^{2 \phi}, e^{2 \phi}, e^{2 \phi}, e^{-8 \phi}\right)
$$

and the coordinates on $S^{4}$ given by

$\mu^{M}=\left(\mu^{i}, \mu^{5}\right)=\left(\sin \xi \hat{\mu}^{i}, \cos \xi\right), \quad i=1,2,3,4$,

with $\hat{\mu}^{i}$ being coordinates on $S^{3}$ satisfying $\hat{\mu}^{i} \hat{\mu}^{i}=1$. With the formulas given in Appendix B, the 11-dimensional metric and the four-form field strength are given by

$$
\begin{aligned}
d \hat{s}_{11}^{2}= & \Delta^{\frac{1}{3}}\left(e^{2 U(r)} d s_{M_{3}}^{2}+e^{2 V(r)} d r^{2}+e^{2 W(r)} d s_{S^{3}}^{2}\right) \\
& +\frac{16}{g^{2}} \Delta^{-\frac{2}{3}}\left[e^{-8 \phi} \sin ^{2} \xi d \xi^{2}\right. \\
& \left.+e^{2 \phi}\left(\cos ^{2} \xi d \xi^{2}+\sin ^{2} \xi d \Omega_{(3)}^{2}\right)\right] \\
\hat{F}_{(4)}= & \frac{64}{g^{3}} \Delta^{-2} \sin ^{4} \xi\left(\mathcal{U} \sin \xi d \xi-10 e^{6 \phi} \phi^{\prime} \cos \xi d r\right) \wedge \epsilon_{(3)} \\
- & 2 \cos \xi e^{8 \phi}\left(k e^{3 W+V-3 U} d r \wedge \operatorname{vol}_{S^{3}}\right. \\
- & \left.l e^{3 U+V-3 W} d r \wedge \operatorname{vol}_{M_{3}}\right) \\
- & \frac{8}{g} \sin \xi\left(k \operatorname{vol}_{M_{3}}+l \operatorname{vol}_{S^{3}}\right) \wedge d \xi
\end{aligned}
$$

with $d \Omega_{(3)}^{2}=d \hat{\mu}^{i} d \hat{\mu}^{i}$ being the metric on a unit $S^{3}$ and 


$$
\begin{aligned}
\Delta & =e^{8 \phi} \cos ^{2} \xi+e^{-2 \phi} \sin ^{2} \xi, \\
\epsilon_{(3)} & =\frac{1}{3 !} \epsilon_{i j k l} \hat{\mu}^{i} d \hat{\mu}^{j} \wedge d \hat{\mu}^{k} \wedge d \hat{\mu}^{l}, \\
\mathcal{U} & =\left(e^{16 \phi}-4 e^{6 \phi}\right) \cos ^{2} \xi-\left(e^{6 \phi}+2 e^{-4 \phi}\right) \sin ^{2} \xi .
\end{aligned}
$$

The $S O(4)$ residual symmetry of the seven-dimensional solution is the isometry of the $S^{3}$ inside the $S^{4}$. The 3-manifold $M_{3}$ can be $M k w_{3}$ or $\mathrm{AdS}_{3}$. Because of the dyonic profile of the four-form field strength, this solution should describe a bound state of M2- and M5-branes similar to the solutions considered in [12]. It is also interesting to find a relation between the solution with $M_{3}=\mathrm{AdS}_{3}$ and the $S O(2,2) \times S O(4) \times S O(4)$ symmetric solution studied in [32].

We can repeat a similar procedure for the $S O(3)$ symmetric solutions. With the index $M=(a, 4,5), a=1,2,3$, the $S L(5) / S O(5)$ scalar matrix is given by

$$
\mathcal{M}=\left(\begin{array}{cc}
e^{4 \phi_{1}} \mathbf{I}_{3} & 0 \\
0 & e^{-6 \phi_{1}} M_{2}
\end{array}\right)
$$

with the $2 \times 2$ matrix $M_{2}$ given by

$$
M_{2}=\left(\begin{array}{cc}
e^{2 \phi_{3}} \cosh ^{2} \phi_{2}+\sinh ^{2} \phi_{2} & \sinh \phi_{2} \cosh \phi_{2}\left(1+e^{-2 \phi_{3}}\right) \\
\sinh \phi_{2} \cosh \phi_{2}\left(1+e^{2 \phi_{3}}\right) & e^{-2 \phi_{3}} \cosh ^{2} \phi_{2}+\sinh ^{2} \phi_{2}
\end{array}\right) .
$$

We now separately discuss the uplifted solutions for the two cases with $\phi_{2}=0$ and $\phi_{3}=0$. We will also denote $k_{5}$ and $l_{5}$ simply by $k$ and $l$ with $k_{4}=\tanh \phi_{2} k$ and $l_{4}=\tanh \phi_{2} l$. Recall also that for $S O(3)$ symmetric solutions, we only have $M_{3}=\mathrm{AdS}_{3}$.

For $\phi_{2}=0$ and the $S^{4}$ coordinates

$$
\mu^{M}=\left(\cos \xi \hat{\mu}^{a}, \sin \xi \cos \psi, \sin \xi \sin \psi\right)
$$

with $\hat{\mu}^{a} \hat{\mu}^{a}=1$, we find the 11-dimensional metric

$$
\begin{aligned}
d \hat{s}_{11}^{2}= & \Delta^{\frac{1}{3}}\left(e^{2 U} d s_{\mathrm{AdS}_{3}}^{2}+e^{2 V} d r^{2}+e^{2 W} d s_{S^{3}}^{2}\right)+\frac{16}{g^{2}} \Delta^{-\frac{2}{3}}\left[e ^ { 4 \phi _ { 1 } } \left(\sin ^{2} \xi d \xi^{2}\right.\right. \\
& \left.+\cos ^{2} \xi d \hat{\mu}^{a} d \hat{\mu}^{a}\right)+e^{-6 \phi_{1}}\left\{\sin ^{2} \xi\left(e^{2 \phi_{3}} \sin ^{2} \psi+e^{-2 \phi_{3}} \cos ^{2} \psi\right) d \psi^{2}\right. \\
& \left.\left.-\sin 2 \psi \sin 2 \xi \sinh 2 \phi_{3} d \xi d \psi+\cos ^{2} \xi\left(e^{2 \phi_{3}} \cos ^{2} \psi+e^{-2 \phi_{3}} \sin ^{2} \psi\right) d \xi^{2}\right\}\right]
\end{aligned}
$$

where

$$
\Delta=e^{-4 \phi_{1}} \cos ^{2} \xi+e^{6 \phi_{1}} \sin ^{2} \xi\left(e^{-2 \phi_{3}} \cos ^{2} \psi+e^{2 \phi_{3}} \sin ^{2} \psi\right) .
$$

The four-form field strength is given by

$$
\begin{aligned}
& \hat{F}_{(4)}=-2 e^{6 \phi_{1}+2 \phi_{3}} \sin \xi \sin \psi d r \wedge\left(k e^{3 W+V-3 U} \operatorname{vol}_{S^{3}}-l e^{3 U+V-3 W} \operatorname{vol}_{\mathrm{AdS}_{3}}\right) \\
& +\frac{8}{g}\left(k \operatorname{vol}_{\mathrm{AdS}_{3}}+l \operatorname{vol}_{S^{3}}\right) \wedge(\cos \xi \sin \psi d \xi+\sin \xi \cos \psi d \psi) \\
& -\frac{64}{g^{3}} \Delta^{-2} \epsilon_{(2)} \wedge\left[\cos ^{2} \xi \sin \xi \mathcal{U} d \xi \wedge d \psi+\phi_{3}^{\prime} e^{12 \phi_{1}} \sin ^{3} \xi \cos ^{2} \xi \sin 2 \psi d r \wedge d \xi\right. \\
& -e^{2 \phi_{1}-2 \phi_{3}} \sin \xi \cos ^{3} \xi d r \wedge\left\{\left(6 \phi_{1}^{\prime} \sin \xi+2 \phi_{3}^{\prime} \sin \xi \cos \psi\right) d \psi-2 \phi_{3}^{\prime} \cos \xi\right. \\
& \times \sin \psi d \xi\}-2 \phi_{1}^{\prime} e^{2 \phi_{1}} \sin 2 \xi \cos ^{2} \xi d r \wedge\left\{\left(e^{-2 \phi_{3}}-e^{2 \phi_{3}}\right) \sin \psi \cos \psi \cos \xi d \xi\right. \\
& \left.\left.+\sin \xi\left(e^{2 \phi_{3}} \sin ^{2} \psi+e^{-2 \phi_{3}} \cos ^{2} \psi\right) d \psi\right\}\right]
\end{aligned}
$$

with

$$
\begin{gathered}
\epsilon_{(2)}=\frac{1}{2} \epsilon_{a b c} \hat{\mu}^{a} d \hat{\mu}^{b} \wedge \hat{\mu}^{c}, \\
\mathcal{U}=\frac{1}{2} e^{2 \phi_{1}}\left[\sin ^{2} \xi\left(1-e^{-4 \phi_{3}}\right)\left\{3 e^{2 \phi_{3}} \cos 2 \psi-e^{10 \phi_{1}}\left(1+\cos 2 \psi-2 e^{4 \phi_{3}} \sin ^{2} \psi\right)\right\}\right. \\
\left.+(\cos 2 \xi-5) \cosh 2 \phi_{3}\right]-e^{-8 \phi_{1}} \cos ^{2} \xi .
\end{gathered}
$$


For $\phi_{3}=0$, we find

$$
\begin{aligned}
d \hat{s}_{11}^{2}= & \Delta^{\frac{1}{3}}\left(e^{2 U} d s_{\mathrm{AdS}_{3}}^{2}+e^{2 V} d r^{2}+e^{2 W} d s_{S^{3}}^{2}\right)+\frac{16}{g^{2}} \Delta^{-\frac{2}{3}}\left[e ^ { 4 \phi _ { 1 } } \left(\sin ^{2} \xi d \xi^{2}\right.\right. \\
& \left.+\cos ^{2} \xi d \hat{\mu}^{a} d \hat{\mu}^{a}\right)+e^{-6 \phi_{1}} \sinh 2 \phi_{2}\left\{\sin 2 \psi\left(\cos ^{2} \xi d \xi^{2}-\sin ^{2} \xi d \psi^{2}\right)\right. \\
& \left.+\sin 2 \xi \cos 2 \psi d \psi d \xi\}+e^{-6 \phi_{1}} \cosh 2 \phi_{2}\left(\cos ^{2} \xi d \xi^{2}+\sin ^{2} \xi d \psi^{2}\right)\right]
\end{aligned}
$$

and

$$
\begin{aligned}
& \hat{F}_{(4)}=2 \sin \xi e^{6 \phi_{1}+V}\left(\cos \psi \tanh \phi_{2}-\sin \psi\right) d r \wedge\left(k e^{3 W-3 U} \operatorname{vol}_{S^{3}}-l e^{3 U-3 W} \operatorname{vol}_{\mathrm{AdS}_{3}}\right) \\
& +\frac{8}{g}\left(k \mathrm{vol}_{\mathrm{AdS}_{3}}+l \mathrm{vol}_{S^{3}}\right) \wedge\left[\left(\tanh \phi_{2} \cos \psi+\sin \psi\right) \cos \xi d \xi\right. \\
& \left.+\sin \xi\left(\cos \psi-\tanh \phi_{2} \sin \psi\right)\right]-\frac{64}{g^{3}} \mathcal{U} \Delta^{-2} \sin \xi \cos ^{2} \xi \epsilon_{(2)} \wedge d \xi \wedge d \psi \\
& +\frac{64}{g^{3}} \Delta^{-2} d r \wedge \epsilon_{(2)} \wedge\left[\frac{1}{2} e^{12 \phi_{1}} \phi_{2}{ }^{\prime} \sin \xi \sin ^{2} 2 \xi \cos 2 \psi d \xi\right. \\
& +\frac{1}{2} e^{-4 \phi_{1}} \cos ^{2} \xi \sin 2 \xi\left\{\sin ^{2} \xi\left(e^{6 \phi_{1}} \cosh 2 \phi_{2}\right)^{\prime} d \psi\right. \\
& \left.+\left(e^{6 \phi_{1}} \sinh 2 \phi_{2}\right)^{\prime}(\cos \xi \cos 2 \psi d \xi-\sin \xi \sin 2 \psi d \psi)\right\} \\
& +2 \phi_{1}{ }^{\prime} e^{2 \phi_{1}} \cos ^{2} \xi \sin 2 \xi\left\{\sin \xi \cosh 2 \phi_{2} d \psi\right. \\
& \left.\left.-\sinh 2 \phi_{2}(\sin \xi \sin 2 \psi d \psi-\cos 2 \psi d \xi)\right\}\right] \text {, }
\end{aligned}
$$

where

$$
\begin{gathered}
\Delta=e^{-4 \phi_{1}} \cos ^{2} \xi+e^{6 \phi_{1}} \sin ^{2} \xi\left(\cosh 2 \phi_{2}-\sin 2 \psi \sinh 2 \phi_{2}\right), \\
\mathcal{U}=\sin ^{2} \xi\left[3 e^{2 \phi_{1}} \sin 2 \psi \sinh 2 \phi_{2}+e^{12 \phi_{1}}\left(6 \cosh ^{2} 2 \phi_{2}-\sin 2 \psi \sinh 4 \phi_{2}\right)\right] \\
+\left(2 e^{-4 \phi_{1}}-3 e^{-8 \phi_{1}}\right) \cos ^{2} \xi+\frac{1}{2} e^{2 \phi_{1}} \cosh 2 \phi_{2}(\cos 2 \xi-5) .
\end{gathered}
$$

All of these solutions should describe bound states of M2- and M5-branes with different transverse spaces and are expected to be holographically dual to conformal surface defects in $N=(2,0)$ SCFT in six dimensions. Solutions with $S O(2) \times S O(2)$ symmetry can similarly be uplifted, but we will not give them here due to their complexity.

\section{Uplift to type IIA theory}

We now carry out a similar analysis for solutions in $\operatorname{CSO}(4,0,1)$ gauge group to find uplifted solutions in tendimensional type IIA theory. Relevant formulas are reviewed in Appendix B. In the solutions we will consider, gauge fields, massive three-forms, and axions $b_{i}=\chi_{i}$ vanish. The ten-dimensional fields are then given only by the metric, the dilaton, and the Neveu Schwarz-Neveu Schwarz (NS-NS) two-form field. Therefore, in this case, we expect the solutions to describe bound states of NS5branes and the fundamental strings.
We begin with a simpler $S O(4)$ symmetric solution in which the $S L(4) / S O(4)$ scalar matrix is given by $\widetilde{\mathcal{M}}_{i j}=\delta_{i j}$. The ten-dimensional metric, the NS-NS three-form flux, and the dilaton are given by

$$
\begin{aligned}
d \hat{s}_{10}^{2} & =e^{\frac{3}{2} \phi_{0}}\left(e^{2 U} d s_{M_{3}}^{2}+e^{2 V} d r^{2}+e^{2 W} d s_{S^{3}}^{2}\right)+\frac{16}{g^{2}} e^{-\frac{5}{2} \phi_{0}} d \Omega_{(3)}^{2}, \\
\hat{H}_{(3)} & =\frac{128}{g^{3}} \epsilon_{(3)}+\frac{8}{g}\left(k \operatorname{vol}_{M_{3}}+l \operatorname{vol}_{S^{3}}\right), \\
\hat{\varphi} & =5 \phi_{0} .
\end{aligned}
$$

It should be noted that, in this case, we have a constant NSNS flux.

For $S O(3)$ symmetric solutions, we parametrize the $S L(4) / S O(4)$ scalar matrix as

$$
\widetilde{\mathcal{M}}_{i j}=\operatorname{diag}\left(e^{2 \phi}, e^{2 \phi}, e^{2 \phi}, e^{-6 \phi}\right)
$$


and choose the $S^{3}$ coordinates to be

$$
\mu^{i}=\left(\sin \xi \hat{\mu}^{a}, \cos \xi\right), \quad a=1,2,3,
$$

with $\hat{\mu}^{a}$ being the coordinates on $S^{2}$ subject to the condition $\hat{\mu}^{a} \hat{\mu}^{a}=1$. We again recall that only solutions with $\phi_{2}=0$ are possible in this case.

With all these ingredients and writing $k=k_{5}$ and $l=l_{5}$, we find that the ten-dimensional fields are given by

$$
\begin{aligned}
& d \hat{s}_{10}^{2}= e^{\frac{3}{2} \phi_{0}} \Delta^{\frac{1}{4}}\left(e^{2 U} d s_{\mathrm{AdS}_{3}}^{2}+e^{2 V} d r^{2}+e^{2 W} d s_{S^{3}}^{2}\right) \\
&+\frac{16}{g^{2}} e^{-\frac{5}{2} \phi_{0}} \Delta^{-\frac{3}{4}}\left[\left(e^{-6 \phi} \sin ^{2} \xi+e^{2 \phi} \cos ^{2} \xi\right) d \xi^{2}\right. \\
&\left.+\sin ^{2} \xi e^{2 \phi} d \hat{\mu}^{a} d \hat{\mu}^{a}\right], \\
& e^{2 \hat{\varphi}}=\Delta^{-1} e^{10 \phi_{0}}, \\
& \hat{H}_{(3)}=\frac{64}{g^{3}} \Delta^{-2} \sin ^{3} \xi\left(\mathcal{U} \sin \xi d \xi+8 e^{4 \phi} \cos \xi \phi^{\prime} d r\right) \\
& \wedge \epsilon_{(2)}+\frac{8}{g}\left(k \operatorname{vol}_{\mathrm{AdS}_{3}}+l \operatorname{vol}_{S^{3}}\right),
\end{aligned}
$$

in which

$$
\begin{aligned}
& \Delta=e^{6 \phi} \cos ^{2} \xi+e^{-2 \phi} \sin ^{2} \xi, \quad \epsilon_{(2)}=\frac{1}{2} \epsilon_{a b c} \hat{\mu}^{a} d \hat{\mu}^{b} \wedge d \hat{\mu}^{c} \\
& \mathcal{U}=e^{12 \phi} \cos ^{2} \xi-e^{-4 \phi} \sin ^{2} \xi-e^{4 \phi}\left(\sin ^{2} \xi+3 \cos ^{2} \xi\right) .
\end{aligned}
$$

The solutions for $\phi_{0}$ and $\phi$ are obtained from $\phi_{1}$ and $\phi_{3}$ in Sec. III B by the following relations:

$\phi=\frac{1}{4}\left(5 \phi_{1}-\phi_{3}\right) \quad$ and $\quad \phi_{0}=-\frac{1}{4}\left(\phi_{3}+3 \phi_{1}\right)$.

These are obtained by comparing the scalar matrices obtained from (137) and (B10).

\section{SUPERSYMMETRIC SOLUTIONS FROM GAUGINGS IN $\overline{40}$ REPRESENTATION}

In this section, we repeat the same analysis for gaugings from $\overline{\mathbf{4 0}}$ representation. Setting $Y_{M N}=0$, we are left with the quadratic constraint

$$
\epsilon_{M R S T U} Z^{R S, N} Z^{T U, P}=0 .
$$

Following [23], we can solve this constraint by taking

$$
Z^{M N, P}=v^{[M} w^{N] P}
$$

with $w^{M N}=w^{(M N)}$ and $v^{M}$ being a five-dimensional vector.

The $S L(5)$ symmetry can be used to fix the vector $v^{M}=\delta_{5}^{M}$. Therefore, it is useful to split the $S L(5)$ index as
$M=(i, 5)$. Setting $w^{55}=w^{i 5}=0$ for simplicity, we can use the remaining $S L(4) \subset S L(5)$ symmetry to diagonalize $w^{i j}$ as

$$
w^{i j}=\operatorname{diag}(\underbrace{1, \ldots, 1}_{p}, \underbrace{-1, \ldots,-1}_{q}, \underbrace{0, \ldots, 0}_{r}) .
$$

The resulting gauge generators read

$$
\left(X_{i j}\right)_{k}^{l}=2 \epsilon_{i j k m} w^{m l}
$$

corresponding to a $\operatorname{CSO}(p, q, r)$ gauge group with $p+q+r=4$.

With the split of $S L(5)$ index $M=(i, 5)$ and the decomposition $S L(5) \rightarrow S L(4) \times S O(1,1)$, we can parametrize the $S L(5) / S O(5)$ coset representative in terms of the $S L(4) / S O(4)$ one as

$$
\mathcal{V}=e^{b_{i} t^{i}} \tilde{\mathcal{V}} e^{\phi_{0} t_{0}}
$$

$\tilde{\mathcal{V}}$ is the $S L(4) / S O(4)$ coset representative, and $t_{0}, t^{i}$ refer to $S O(1,1)$ and four nilpotent generators, respectively. The unimodular matrix $\mathcal{M}_{M N}$ is then given by

$$
\mathcal{M}_{M N}=\left(\begin{array}{cc}
e^{-2 \phi_{0}} \tilde{\mathcal{M}}_{i j}+e^{8 \phi_{0}} b_{i} b_{j} & e^{8 \phi_{0}} b_{i} \\
e^{8 \phi_{0}} b_{j} & e^{8 \phi_{0}}
\end{array}\right)
$$

with $\tilde{\mathcal{M}}_{i j}=\left(\tilde{\mathcal{V}} \tilde{\mathcal{V}}^{T}\right)_{i j}$. Using (25), we can compute the scalar potential for these gaugings

$$
\begin{aligned}
\mathbf{V}= & \frac{g^{2}}{4} e^{14 \phi_{0}} b_{i} w^{i j} \tilde{\mathcal{M}}_{j k} w^{k l} b_{l} \\
& +\frac{g^{2}}{4} e^{4 \phi_{0}}\left(2 \tilde{\mathcal{M}}_{i j} w^{j k} \tilde{\mathcal{M}}_{k l} w^{l i}-\left(\tilde{\mathcal{M}}_{i j} w^{i j}\right)^{2}\right) .
\end{aligned}
$$

The presence of the dilaton prefactor $e^{\phi_{0}}$ shows that this potential does not admit any critical points. Note also that we can always consistently set the nilpotent scalars $b_{i}$ to zero for simplicity since they do not appear linearly in any terms in the Lagrangian.

We will use the same ansatz as in the case of gaugings in the 15 representation to find charged domain wall solutions. However, we note here that, for gaugings in the $\overline{\mathbf{4 0}}$ representation, there are no massive three-form fields $S_{\mu \nu \rho}^{M}$. The three-form fluxes given in (52) in this case correspond solely to the two-form fields $B_{\mu \nu M}$. We now consider a number of possible solutions with different symmetries.

\section{A. $S O(4)$ symmetric charged domain walls}

For $S O(4)$ residual symmetry under which only the scalar field $\phi_{0}$ is invariant, we have $\widetilde{\mathcal{M}}_{i j}=\delta_{i j}$. The only gauge group that can accommodate the $S O(4)$ unbroken symmetry is $S O(4)$ with the embedding tensor component 
$w^{i j}=\delta^{i j}$. The scalar potential as obtained from (213) takes a very simple form

$$
\mathbf{V}=-2 g^{2} e^{4 \phi_{0}}
$$

which does not admit any critical points. We will consider solutions with nonvanishing $\mathcal{H}_{\mu \nu \rho 5}^{(3)}$ which is an $S O(4)$ singlet.

In this $S O(4)$ gauging, there are four massive two-form fields $B_{\mu \nu i}, i=1, \ldots, 4$, and one massless two-form field $B_{\mu \nu 5}$ with the latter being an $S O(4)$ singlet. We will take the ansatz for $B_{\mu \nu 5}$ as given in (60). With the following projection conditions:

$$
\gamma_{\hat{3}} \epsilon_{0}^{a}=-\left(\Gamma_{5}\right)^{a}{ }_{b} \epsilon_{0}^{b}=\epsilon_{0}^{a},
$$

the BPS equations are given by

$$
\begin{gathered}
U^{\prime}=W^{\prime}=\frac{1}{5} e^{V}\left(2 e^{-2 \phi_{0}} g \sec 2 \theta-e^{-U} \tau \tan 2 \theta\right), \\
\phi_{0}^{\prime}=\frac{1}{10} e^{V}\left(2 e^{-2 \phi_{0}} g \sec 2 \theta-e^{-U} \tau \tan 2 \theta\right), \\
k=-\frac{1}{2} e^{2 U-4 \phi_{0}} \tau, \theta^{\prime}=0 \\
l=-\frac{1}{2} e^{2 U-4 \phi_{0}} \tau \sec 2 \theta+3 e^{3 U-6 \phi_{0}} g \tan 2 \theta
\end{gathered}
$$

together with an algebraic constraint

$$
\kappa=\tau \sec 2 \theta-2 e^{U-2 \phi_{0}} g \tan 2 \theta
$$

In this case, we find that $\theta$ is constant. Choosing $V=0$, we find the following solution:

$$
\begin{gathered}
U=W=2 \phi_{0}, \\
e^{2 \phi_{0}}=\frac{2}{5} g r \sec 2 \theta-\frac{1}{5} \tau r \tan 2 \theta+C, \\
k=-\frac{1}{2} \tau, \\
l=-\frac{1}{2} \tau \sec 2 \theta+g \tan 2 \theta
\end{gathered}
$$

with an integration constant $C$. For a particular value of $\theta=0$, we find the solution

$$
U=W=2 \phi_{0}, \quad e^{2 \phi_{0}}=\frac{2}{5} g r+C, \quad k=l=-\frac{1}{2} \tau .
$$

\section{Coupling to $\mathrm{SO}(3)$ gauge fields}

We now consider charged domain wall solutions with nonvanishing $S O(3) \subset S O(4)$ gauge fields. In this case, the projector $\left(\Gamma_{5}\right)^{a}{ }_{b} \epsilon_{0}^{b}=-\epsilon_{0}^{a}$ implies that the nonvanishing gauge fields correspond to the self-dual $S O(3) \subset S O(4)$ given by

$$
\begin{aligned}
& A_{(1)}^{23}=A_{(1)}^{14}=\frac{\kappa}{16} p(r) e^{-W(r)} e^{\hat{4}}, \\
& A_{(1)}^{31}=A_{(1)}^{24}=\frac{\kappa}{16} p(r) e^{-W(r)} e^{\hat{5}}, \\
& A_{(1)}^{12}=A_{(1)}^{34}=\frac{\kappa}{16} p(r) e^{-W(r)} e^{\hat{6}} .
\end{aligned}
$$

The two-form field strengths are straightforward to obtain

$$
\begin{aligned}
& F_{(2)}^{12}=F_{(2)}^{34}=e^{-V-W} \frac{\kappa}{16} p^{\prime} e^{\hat{3}} \wedge e^{\hat{6}}+e^{-2 W} \frac{\kappa^{2}}{32} p(2-g p) e^{\hat{4}} \wedge e^{\hat{5}} \\
& F_{(2)}^{23}=F_{(2)}^{14}=e^{-V-W} \frac{\kappa}{16} p^{\prime} e^{\hat{3}} \wedge e^{\hat{4}}+e^{-2 W} \frac{\kappa^{2}}{32} p(2-g p) e^{\hat{5}} \wedge e^{\hat{6}} \\
& F_{(2)}^{31}=F_{(2)}^{24}=e^{-V-W} \frac{\kappa}{16} p^{\prime} e^{\hat{3}} \wedge e^{\hat{5}}+e^{-2 W} \frac{\kappa^{2}}{32} p(2-g p) e^{\hat{6}} \wedge e^{\hat{4}}
\end{aligned}
$$

Since the components of the embedding tensor $Z^{i j, 5}$ vanish, the two-form field $B_{\mu \nu 5}$ does not contribute to the modified two-form field strengths. Imposing the projection conditions (125) and (215), we find the following BPS equations:

$$
\begin{aligned}
U^{\prime}= & \frac{e^{V-2\left(W+\phi_{0}\right)}}{80 \cos 2 \theta}\left[16 e^{2 W}\left(g(3 \cos 4 \theta-1)+2 e^{2 \phi_{0}-U} \tau \sin 2 \theta\right)\right. \\
& \left.-3 e^{4 \phi_{0}}\left(\kappa^{2} p(g p-2)(\cos 4 \theta-3)-8 e^{W-2 \phi_{0}} \kappa(g p-1) \sin 4 \theta\right)\right], \\
W^{\prime}= & \frac{e^{V-2\left(W+\phi_{0}\right)}}{40 \cos 2 \theta}\left[8 e^{2 W}\left(2 g(2-\cos 4 \theta)-3 e^{2 \phi_{0}-U} \tau \sin 2 \theta\right)\right. \\
& \left.+e^{4 \phi_{0}}\left(\kappa^{2} p(g p-2)(\cos 4 \theta-8)-8 e^{W-2 \phi_{0}} \kappa(g p-1) \sin 4 \theta\right)\right],
\end{aligned}
$$




$$
\begin{gathered}
\phi_{0}^{\prime}=\frac{e^{V-2\left(W+\phi_{0}\right)}}{160 \cos 2 \theta}\left[16 e^{2 W}\left(g(3 \cos 4 \theta-1)+2 e^{2 \phi_{0}-U} \tau \sin 2 \theta\right)\right. \\
\left.+3 e^{4 \phi_{0}}\left(\kappa^{2} p(g p-2)(3-\cos 4 \theta)+8 e^{W-2 \phi_{0}} \kappa(g p-1) \sin 4 \theta\right)\right], \\
\theta^{\prime}=\frac{e^{V-2\left(W+\phi_{0}\right)}}{16}\left[24 e^{W+2 \phi_{0}}\left(e^{W-U} \tau+\kappa(g p-1) \cos 2 \theta\right)\right. \\
\left.-3\left(16 g e^{2 W}-e^{4 \phi_{0}} \kappa^{2} p(g p-2)\right) \sin 2 \theta\right], \\
k=-\frac{1}{2} e^{2 U-4 \phi_{0}} \tau, \\
l=\frac{1}{8} e^{3 W-6 \phi_{0}}\left[-16 g \tan 2 \theta+8 e^{2 \phi_{0}-U} \tau \sec 2 \theta\right. \\
\left.+3 e^{4 \phi_{0}-2 W}\left(\kappa^{2} p(g p-2) \tan 2 \theta+4 e^{W-2 \phi_{0}} \kappa(g p-1)\right)\right], \\
p^{\prime}=\frac{e^{V-W-4 \phi_{0}}}{2 \kappa}\left[8 e^{W+2 \phi_{0}}\left(e^{W-U} \tau+\kappa(g p-1) \cos 2 \theta\right)\right. \\
\left.\quad-\left(16 g e^{2 W}-e^{4 \phi_{0}} \kappa^{2} p(g p-2)\right) \sin 2 \theta\right] .
\end{gathered}
$$

It can be verified that these BPS equations satisfy the secondorder field equations without any additional constraint.

Since there is not an asymptotically locally $\mathrm{AdS}_{7}$ configuration, we will consider flow solutions from a charged domain wall without vector fields given in (221)-(224) to a singular solution with nonvanishing gauge fields. To find numerical solutions, we will consider the charged domain wall with $\theta=0$ given in (225) for simplicity. As $r \rightarrow-\frac{5 C}{2 g}$, we impose the following boundary conditions:

$$
\begin{aligned}
& U \sim W \sim \ln \left[\frac{2 g r}{5}+C\right], \quad \phi \sim \frac{1}{2} \ln \left[\frac{2 g r}{5}+C\right], \\
& p \sim 0, \quad k \sim l \sim-\frac{\tau}{2}
\end{aligned}
$$

with $\kappa=\tau$. An example of the BPS flows is shown in Fig. 7. From this solution, it can be seen that $k$ is constant along the flow since the above BPS equations give $U^{\prime}=$ $2 \phi_{0}{ }^{\prime}$, which implies the constancy of $U-2 \phi_{0}$. It should

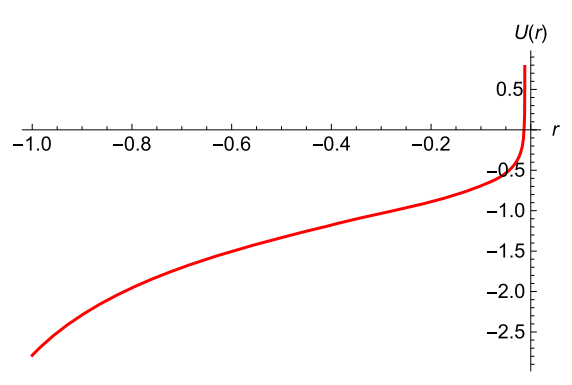

(a) $U$ solution

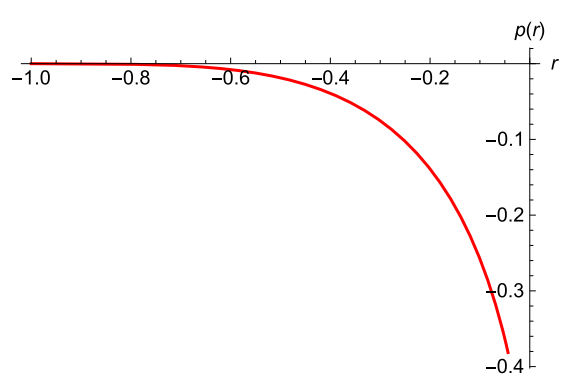

(d) $p$ solution

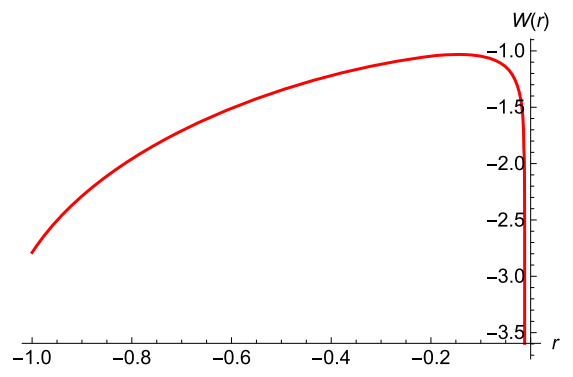

(b) $W$ solution

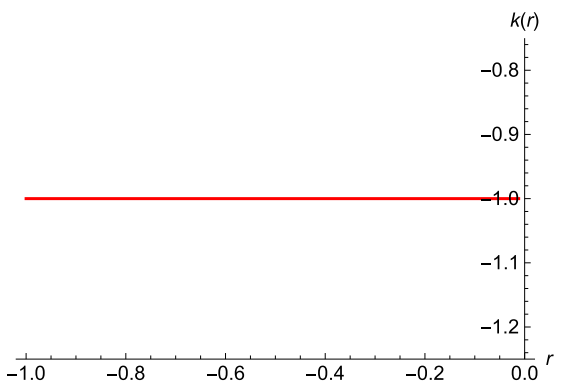

(e) $k$ solution

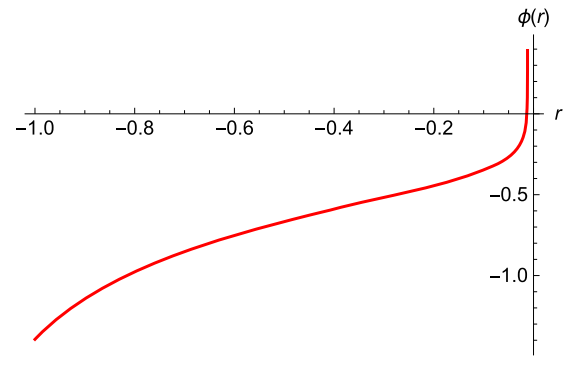

(c) $\phi$ solution

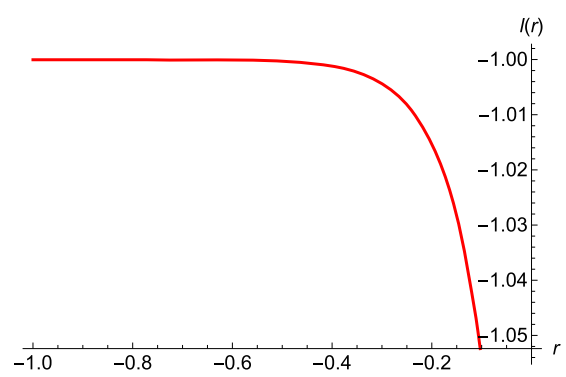

(f) $l$ solution

FIG. 7. A BPS flow from a charged domain wall at $r=-1$ to a singularity at $r=0$ in $S O$ (4) gauge group with $g=1, \kappa=\tau=2$, and $C=\frac{2}{5}$. 
also be noted that this solution is similar to that in $\operatorname{CSO}(4,0,1)$ gauge group given in Fig. 5. We also expect this solution to describe a surface defect within an $N=$ $(2,0)$ nonconformal field theory.

\section{B. $S O(3)$ symmetric charged domain walls}

In this section, we look for more complicated solutions with $S O(3)$ residual symmetry generated by $X_{i j}$ with $i$, $j=1,2,3$. Gauge groups containing an $S O(3)$ subgroup are $S O(4), S O(3,1)$, and $\operatorname{CSO}(3,0,1)$. These gauge groups are described by the embedding tensor $w^{i j}$ of the form

$$
w^{i j}=\operatorname{diag}(+1,+1,+1, \rho)
$$

with $\rho=1,-1,0$, respectively.

Among the ten $S L(4) / S O(4)$ scalars, there is one $S O(3)$ singlet parametrized by the $S L(4) / S O(4)$ coset representative

$$
\tilde{\mathcal{V}}=\operatorname{diag}\left(e^{\phi}, e^{\phi}, e^{\phi}, e^{-3 \phi}\right) .
$$

We then obtain the scalar potential using (213)

$$
\mathbf{V}=-\frac{g^{2}}{4} e^{-4\left(\phi_{0}+3 \phi\right)}\left(3 e^{16 \phi}+6 \rho e^{8 \phi}-\rho^{2}\right) .
$$

To find the BPS equations, we use the same ansatz for the modified three-form field strength (60) and impose the projection conditions (215). We note here that, in this case, there are two two-form fields, $B_{\mu \nu 4}$ and $B_{\mu \nu 5}$, which are $S O(3)$ singlets. For $\operatorname{CSO}(3,0,1)$ gauge group with $\rho=0$, both of them are massless while for the other two gauge groups, the former is massive while the latter is massless. However, in this case, we are not able to consistently incorporate $B_{\mu \nu 4}$ in the BPS equations. We will accordingly restrict ourselves to the solutions with only $B_{\mu \nu 5}$ nonvanishing.

Consistency with the field equations also leads to the conditions given in (142). With all these, the resulting BPS equations are given by

$$
\begin{gathered}
U^{\prime}=W^{\prime}=\frac{g}{10} e^{V-6 \phi-2 \phi_{0}}\left(3 e^{8 \phi_{1}}+\rho\right), \\
\phi_{0}^{\prime}=\frac{g}{20} e^{V-6 \phi-2 \phi_{0}}\left(3 e^{8 \phi_{1}}+\rho\right), \\
\phi^{\prime}=-\frac{g}{4} e^{V-6 \phi-2 \phi_{0}}\left(3 e^{8 \phi_{1}}-\rho\right), \\
k=-\frac{1}{2} e^{3 U-W-4 \phi_{0}} \kappa, \\
l=-\frac{1}{2} e^{2 W-4 \phi_{0}} \kappa .
\end{gathered}
$$

Setting $W=U$ and $V=0$, we find the solutions for $U, \phi_{0}$, $k$, and $l$ as functions of $\phi$,

$$
U=\frac{2}{5} \phi-\frac{1}{5} \ln \left(e^{8 \phi}-\rho\right)
$$

$$
\begin{gathered}
\phi_{0}=\frac{1}{5} \phi-\frac{1}{10} \ln \left(e^{8 \phi}-\rho\right)+C_{0}, \\
k=l=-\frac{1}{2} e^{-4 C_{0}} \kappa,
\end{gathered}
$$

in which $C_{0}$ is an integration constant.

The solution for $\phi(r)$ is given by

$$
\phi=-\frac{5}{16} \ln \left[\frac{4}{5}\left(e^{-2 C_{0}} g r-C_{1}\right)\right]
$$

for $\rho=0$ and

$$
\begin{aligned}
& 4 \operatorname{g\rho } r\left(e^{8 \phi}-\rho\right)^{1 / 5} \\
& \quad=5 e^{2 C_{1}+\frac{32}{5} \phi}\left[4-3\left(1-\rho e^{8 \phi}\right)^{1 / 5}{ }_{2} F_{1}\left(\frac{1}{5}, \frac{4}{5}, \frac{9}{5}, \rho e^{8 \phi}\right)\right]
\end{aligned}
$$

for $\rho= \pm 1$. In the last equation, ${ }_{2} F_{1}$ is the hypergeometric function. This solution is again the domain wall found in [26] with a nonvanishing three-form flux.

As in the $S O(3)$ symmetric solutions from the gaugings in the 15 representation, coupling to $S O(3)$ vector fields does not lead to new solutions. Consistency with the field equations implies either vanishing two-form fields or vanishing gauge fields. We also note that repeating the same analysis for $S O(2) \times S O(2)$ and $S O(2)$ symmetric solutions leads to the domain wall solutions given in [26] with a constant three-form flux

$$
k=l=-\frac{1}{2} \tau .
$$

We will not give further detail for these cases to avoid a repetition.

\section{SUPERSYMMETRIC SOLUTIONS FROM GAUGINGS IN 15 AND 40 REPRESENTATIONS}

In this section, we consider gaugings with both components of the embedding tensor in $\mathbf{1 5}$ and $\overline{\mathbf{4 0}}$ representations nonvanishing. We first give a brief review of these gaugings as constructed in [23]. A particular basis can be chosen such that nonvanishing components of the embedding tensor are given by

$$
Y_{x y}, \quad Z^{x \alpha, \beta}=Z^{x(\alpha, \beta)}, \quad Z^{\alpha \beta, \gamma}
$$


with $x=1, \ldots, t$ and $\alpha=t+1, \ldots, 5$. The $S L(5)$ index $M, N, \ldots$, are then split into $(x, \alpha)$.

In terms of these components, the quadratic constraint (14) reads

$$
Y_{x y} Z^{y \alpha, \beta}+2 \epsilon_{x M N P Q} Z^{M N, \alpha} Z^{P Q, \beta}=0 .
$$

$Y_{x y}$ is chosen to be

$$
Y_{x y}=\operatorname{diag}(\underbrace{1, \ldots, 1}_{p}, \underbrace{-1, \ldots,-1}_{q}) .
$$

We will consider two gauge groups, namely $S O(2,1) \ltimes$ $\mathbf{R}^{4}$ and $S O(2) \ltimes \mathbf{R}^{4}$ given in [23]. The latter can also be obtained from the Scherk-Schwarz reduction of the maximal gauged supergravity in eight dimensions.

We begin with the $t=3$ case in which $Y_{x y}=$ $\operatorname{diag}(1,1,-1)$ corresponding to $S O(2,1) \ltimes \mathbf{R}^{4}$ gauge group. The corresponding gauge generators are given by

$$
X_{M}^{N}=\left(\begin{array}{cc}
\lambda^{z}\left(t^{z}\right)_{x}^{y} & Q_{x}^{(4) \beta} \\
0_{2 \times 3} & \frac{1}{2} \lambda^{z}\left(\zeta^{z}\right)_{\alpha}^{\beta}
\end{array}\right)
$$

with $\lambda^{z} \in \mathbb{R}$ and $\left(t^{z}\right)_{x}{ }^{y}=\epsilon^{z y u} Y_{u x}$ being generators of $S O(2,1)$ in the adjoint representation. The nilpotent generators $Q_{x}^{(4) \alpha}$ transform as $\mathbf{4}$ under $S O(2,1)$. In terms of $\zeta^{x}$, the component $Z^{x \alpha, \beta}$ of the embedding tensor takes the form

$$
Z^{x \alpha, \beta}=-\frac{1}{16} \epsilon^{\alpha \gamma}\left(\zeta^{x}\right)_{\gamma}^{\beta} .
$$

The explicit form of $\zeta^{x}$ can be given in terms of Pauli matrices as

$$
\zeta^{1}=\sigma_{1}, \quad \zeta^{2}=\sigma_{3}, \quad \zeta^{3}=i \sigma_{2}
$$

We now consider charged domain wall solutions with $S O(2) \subset S O(2,1)$ symmetry. As shown in [26], there are four $S O(2)$ singlet scalars corresponding to the following noncompact generators:

$$
\begin{aligned}
& \bar{Y}_{1}=2 e_{1,1}+2 e_{2,2}+2 e_{3,3}-3 e_{4,4}-3 e_{5,5}, \\
& \bar{Y}_{2}=e_{1,1}+e_{2,2}-2 e_{3,3}, \\
& \bar{Y}_{3}=e_{1,4}+e_{2,5}+e_{4,1}+e_{5,2}, \\
& \bar{Y}_{4}=e_{1,5}-e_{2,4}-e_{4,2}+e_{5,1} .
\end{aligned}
$$

The $S L(5) / S O(5)$ coset representative can be written as

$$
\mathcal{V}=e^{\phi_{1} \bar{Y}_{1}+\phi_{2} \bar{Y}_{2}+\phi_{3} \bar{Y}_{3}+\phi_{4} \bar{Y}_{4}} .
$$

The resulting scalar potential is given by

$$
\mathbf{V}=\frac{g^{2}}{64} e^{-2\left(4 \phi_{1}-\phi_{2}\right)}\left[6 \cosh 2 \phi_{3} \cosh 2 \phi_{4}+e^{6 \phi_{2}}\right],
$$

which does not admit any critical points.

We now repeat the same analysis as in the previous sections. We first discuss the three-form fluxes that are singlet under the $S O(2)$ residual symmetry. In the ungauged supergravity, the five two-forms transform as $\mathbf{5}$ under $S L(5)$. From the particular form of the gauge generators given in (257), we can see that the $S O(2)$ symmetry under consideration here is embedded diagonally along the $1,2,4,5$ directions. Under $S O(2) \times S O(2) \subset S O(5) \subset S L(5)$, the two-forms transform as $(\mathbf{1}, \mathbf{1})+(\mathbf{1}, \mathbf{2})+(\mathbf{2}, \mathbf{1})$. Under $S O(2)=[S O(2) \times S O(2)]_{\text {diag }}$, these two-forms transform as $\mathbf{1}+\mathbf{2}+\mathbf{2}$. Therefore, there is only one singlet two-form field under the $S O(2)$ unbroken symmetry. In gauged supergravity, this two-form field will be gauged away by a three-form gauge transformation due to the nonvanishing component $Y_{33}$ of the embedding tensor. The $S O(2)$ singlet is then described by a massive three-form field $S_{\mu \nu \rho}^{3}$.

We will take the ansatz for the three-form field strength to be

$$
\begin{aligned}
\mathcal{H}_{\hat{m} \hat{n} \hat{p} 3}^{(3)} & =k(r) e^{-3 U(r)} \varepsilon_{\hat{m} \hat{n} \hat{p}} \quad \text { and } \\
\mathcal{H}_{\hat{i} \hat{j} \hat{k} 3}^{(3)} & =l(r) e^{-3 W(r)} \varepsilon_{\hat{i} \hat{j} \hat{k}} .
\end{aligned}
$$

After imposing the following projection conditions:

$$
\gamma_{\hat{3}} \epsilon_{0}^{a}=-\left(\Gamma_{3}{ }^{a}{ }_{b} \epsilon_{0}^{b}=\epsilon_{0}^{a},\right.
$$

we find the following BPS equations:

$$
\begin{gathered}
U^{\prime}=W^{\prime}=\frac{g}{40} e^{-2\left(2 \phi_{1}+\phi_{2}\right)+V}\left(3 \cosh 2 \phi_{3} \cosh 2 \phi_{4}-e^{6 \phi_{2}}\right), \\
\phi_{1}^{\prime}=\frac{g}{240} e^{-2\left(\phi_{1}+\phi_{2}\right)+V}\left(15 \operatorname{sech} 2 \phi_{3} \operatorname{sech} 2 \phi_{4}-3 \cosh 2 \phi_{3} \cosh 2 \phi_{4}-4 e^{6 \phi_{2}}\right), \\
\phi_{2}^{\prime}=\frac{g}{48} e^{-2\left(\phi_{1}+\phi_{2}\right)+V}\left(3 \operatorname{sech} 2 \phi_{3} \operatorname{sech} 2 \phi_{4}+3 \cosh 2 \phi_{3} \cosh 2 \phi_{4}+4 e^{6 \phi_{2}}\right), \\
\phi_{3}^{\prime}=-\frac{3 g}{16} e^{-2\left(2 \phi_{1}+\phi_{2}\right)+V} \sinh 2 \phi_{3} \operatorname{sech} 2 \phi_{4},
\end{gathered}
$$




$$
\begin{gathered}
\phi_{4}^{\prime}=-\frac{3 g}{16} e^{-2\left(2 \phi_{1}+\phi_{2}\right)+V} \cosh 2 \phi_{3} \sinh 2 \phi_{4}, \\
k=-\frac{1}{2} e^{2 U+2 \phi_{1}-2 \phi_{2}} \tau, \\
l=-\frac{1}{2} e^{3 W-U+2 \phi_{1}-2 \phi_{2}} \tau .
\end{gathered}
$$

In these equations, we have imposed the conditions (142) for consistency.

By choosing $V=4 \phi_{1}+2 \phi_{2}$ and taking $W=U$ for convenience, we obtain a charged domain wall solution

$$
\begin{gathered}
\phi_{1}=\frac{2}{15} \phi_{3}+\frac{1}{5} C_{2}-\frac{1}{60} \ln \left[\frac{9}{16}\left(e^{2 C_{4}}-e^{4 \phi_{3}}-2 e^{2 C_{4}+4 \phi_{3}}+e^{2 C_{4}+8 \phi_{3}}\right)\right] \\
+\frac{1}{10} \ln \left[e^{4 \phi_{3}}+1\right]-\frac{1}{5} \ln \left[e^{4 \phi_{3}}-1\right], \\
\phi_{2}=-5 \phi_{1}+C_{2}+\ln \left[e^{3 \phi_{3}}+1\right]-\ln \left[e^{3 \phi_{3}}-1\right], \\
\phi_{3}=\frac{1}{4} \ln \left[\frac{\left.1+4 e^{2 C_{4}}-2 e^{\frac{3 g r}{8}}+e^{\frac{3 g r}{4}}\right]}{1+4 e^{2 C_{4}}+2 e^{\frac{3 g r}{8}}+e^{\frac{3 g r}{4}}}\right] \\
\phi_{4}=\frac{1}{4} \ln \left[\frac{e^{2 \phi_{3}}-e^{C_{4}}+e^{C_{4}+4 \phi_{3}}}{e^{2 \phi_{3}}+e^{C_{4}}-e^{C_{4}+4 \phi_{3}}}\right] \\
U=-\frac{1}{5} \phi_{3}-\frac{1}{20} C_{2}+\frac{3}{20} \ln \left[e^{2 C_{4}}-e^{4 \phi_{3}}-2 e^{2 C_{4}+4 \phi_{3}}+e^{2 C_{4}+8 \phi_{3}}\right] \\
-\ln \left[\frac{16}{9}\right]-\frac{1}{5} \ln \left[e^{4 \phi_{3}}-1\right], \\
k=l=-\frac{e^{\frac{3}{10}\left(C_{2}+4 \phi_{3}\right)} \tau}{2^{2 / 5} \times 3^{3 / 10}} \frac{\left(e^{2 C_{4}}-e^{4 \phi_{3}}-2 e^{2 C_{4}+4 \phi_{3}}+e^{2 C_{4}+8 \phi_{3}}\right)^{1 / 10}}{\left(e^{4 \phi_{3}}-1\right)^{4 / 5}} .
\end{gathered}
$$

This is just the $\frac{1}{4}$-BPS domain wall obtained in [26] together with the running dyonic profile of the three-form flux. It is useful to emphasize here that this solution is $\frac{1}{4}$-supersymmetric. In general, domain wall solutions from gaugings in both $\mathbf{1 5}$ and $\overline{\mathbf{4 0}}$ representations preserve only $\frac{1}{4}$ of the original supersymmetry; see a general discussion in [33] and explicit solutions in [26]. From the above solution, we see that the solutions with a nonvanishing three-form flux do not break supersymmetry any further.

We end this section by giving a comment on the $t=2$ case with $S O(2) \ltimes \mathbf{R}^{4}$ gauge group. Repeating the same procedure leads to a charged domain wall given by the solution found in [26] with a constant three-form flux given in (253). In contrast to the $t=3$ case, the three-form flux $\mathcal{H}_{\mu \nu \rho 3}^{(3)}$ is due to the massless two-form field $B_{\mu \nu 3}$ since, in this case, we have $Y_{33}=0$. We will not give the full detail of this analysis here as it closely follows that of the previous cases.

\section{CONCLUSIONS AND DISCUSSIONS}

In this paper, we have studied supersymmetric solutions of the maximal gauged supergravity in seven dimensions with various gauge groups. These solutions are charged domain walls with $M_{3} \times S^{3}$ slices, for $M_{3}=M k w_{3}, \mathrm{AdS}_{3}$, and nonvanishing three-form fluxes. All of these solutions can be obtained analytically. For $S O(4)$ residual symmetry, the charged domain wall solutions can couple to $S O(3) \subset$ $S O(4)$ gauge fields, but the corresponding solutions can only be obtained numerically. For $S O(3)$ symmetric solutions, coupling to $S O(3)$ gauge fields does not lead to a consistent set of BPS equations that is compatible with the field equations. In this case, only solutions with either nonvanishing three-form fluxes or nonvanishing gauge fields are possible. Apart from these solutions, we have also given a number of $S O(2) \times S O(2)$ and $S O(2)$ symmetric solutions. 
For $S O(5)$ gauge group, the gauged supergravity admits a supersymmetric $\mathrm{AdS}_{7}$ vacuum dual to an $N=(2,0)$ SCFT in six dimensions. In this case, the solutions with an $\mathrm{AdS}_{3} \times S^{3}$ slice can be interpreted as surface defects within the $N=(2,0)$ SCFT. For other gauge groups, the supersymmetric vacua, with only the metric and scalars nonvanishing, take the form of half-supersymmetric domain walls dual to $N=(2,0)$ nonconformal field theories in six dimensions. We then expect these $\operatorname{AdS}_{3} \times S^{3}$-sliced domain wall solutions to describe $\frac{1}{4}$-BPS surface defects in the dual $N=(2,0)$ quantum field theories. For a number of solutions, we have found that the charged domain walls are simply given by the domain wall solutions given in [26] with constant three-form fluxes. However, the charged domain walls preserve only $\frac{1}{4}$ of the original supersymmetry as opposed to the usual domain walls, which are $\frac{1}{2}$ supersymmetric except for the domain walls from gaugings in both $\mathbf{1 5}$ and $\overline{\mathbf{4 0}}$ representations in which both charged and standard domain walls are $\frac{1}{4}$ supersymmetric.

Both gaugings in $\mathbf{1 5}$ and $\overline{\mathbf{4 0}}$ representations we have studied can, respectively, be uplifted to 11-dimensional supergravity and type IIB theory as shown in $[27,28]$. We have performed only the uplift for solutions in $S O(5)$ and $C S O(4,0,1)$ gauge groups with $S O(4)$ and $S O(3)$ symmetries. In these cases, the complete truncation ansatze of 11-dimensional supergravity on $S^{4}$ and type IIA theory on $S^{3}$ are known. Similar to the solutions in [12], the uplifted solutions in these two gauge groups should describe bound states of M2- and M5-branes and of F1-strings and NS5-branes, respectively. It is natural to extend this study by constructing the full truncation ansatze of 11-dimensional supergravity on $H^{p, q} \circ T^{5-p-q}$ and type IIB theory on $H^{p, q} \circ T^{4-p-q}$. These can be used to uplift the solutions in $C S O(p, q, 5-p-q)$ and $C S O(p, q$, $4-p-q)$ gauge groups for any values of $p$ and $q$ leading to the full holographic interpretation of the sevendimensional solutions found here.

Finding the description of conformal defects, dual to the supergravity solutions given in this paper, in the dual $N=$ $(2,0)$ SCFT and $N=(2,0)$ QFT would be interesting and could provide another verification for the validity of the AdS/CFT correspondence. Finally, finding solutions of the form $\mathrm{AdS}_{d} \times \Sigma^{7-d}$ in seven-dimensional gauged supergravity with various gauge groups is also of particular interest. These solutions would be dual to twisted compactifications of $N=(2,0)$ SCFT and $N=(2,0)$ QFT in six dimensions on a $(7-d)$-manifold $\Sigma^{7-d}$ to $(d-1)$ dimensional SCFT.

\section{ACKNOWLEDGMENTS}

This work is supported by The Thailand Research Fund (TRF) under Grant No. RSA6280022.

\section{APPENDIX A: BOSONIC FIELD EQUATIONS}

In this appendix, we give the explicit form of the bosonic field equations derived from the Lagrangian (22). These equations read

$$
\begin{aligned}
0= & R_{\mu \nu}-\frac{1}{4} \mathcal{M}_{M P} \mathcal{M}_{N Q}\left(D_{\mu} \mathcal{M}^{M N}\right)\left(D_{\nu} \mathcal{M}^{P Q}\right)-\frac{2}{5} g_{\mu \nu} \mathbf{V} \\
& -4 \mathcal{M}_{M P} \mathcal{M}_{N Q}\left(\mathcal{H}_{\mu \rho}^{(2) M N} \mathcal{H}^{(2) P Q}{ }_{\nu}{ }^{2}-\frac{1}{10} g_{\mu \nu} \mathcal{H}_{\rho \sigma}^{(2) M N} \mathcal{H}^{(2) P Q \rho \sigma}\right) \\
& -\mathcal{M}^{M N}\left(\mathcal{H}_{\mu \rho \sigma M}^{(3)} \mathcal{H}_{\nu}^{(3) \rho \sigma}{ }_{N}-\frac{2}{15} g_{\mu \nu} \mathcal{H}_{\rho \sigma \lambda M}^{(3)} \mathcal{H}^{(3) \rho \sigma \lambda}\right) \\
0= & D^{\mu}\left(\mathcal{M}_{M P} D_{\mu} \mathcal{M}^{P N}\right)-\frac{g^{2}}{8} \mathcal{M}^{P Q} \mathcal{M}^{R N}\left(2 Y_{R Q} Y_{P M}-Y_{P Q} Y_{R M}\right) \\
& -\frac{4}{6} \mathcal{M}^{P N} \mathcal{H}_{\mu \nu \rho M}^{(3)} \mathcal{H}^{(3) \mu \nu \rho}{ }_{P}-8 \mathcal{M}_{M P} \mathcal{M}_{Q R} \mathcal{H}_{\mu \nu}^{(2) P Q} \mathcal{H}^{(2) R N \mu \nu} \\
+ & 4 g^{2} Z^{Q T, P} Z^{N R, S} \mathcal{M}_{Q M}\left(2 \mathcal{M}_{T R} \mathcal{M}_{P S}-\mathcal{M}_{T P} \mathcal{M}_{R S}\right) \\
+ & 4 g^{2} Z^{Q T, P} Z^{R S, N} \mathcal{M}_{Q S}\left(2 \mathcal{M}_{T P} \mathcal{M}_{R M}-\mathcal{M}_{T R} \mathcal{M}_{P M}\right) \\
& -4 g^{2} \delta_{M}^{N} Z^{T U, P} Z^{Q R, S} \mathcal{M}_{T Q}\left(\mathcal{M}_{U R} \mathcal{M}_{P S}-\mathcal{M}_{U P} \mathcal{M}_{R S}\right) \\
+ & \frac{8}{5} \delta_{M}^{N}\left(\mathbf{V}+\mathcal{M}_{S P} \mathcal{M}_{Q R} \mathcal{H}_{\mu \nu}^{(2) P Q} \mathcal{H}^{(2) R S \mu \nu}+\frac{1}{16} \mathcal{M}^{P Q} \mathcal{H}_{\mu \nu \rho P}^{(3)} \mathcal{H}^{(3) \mu \nu \rho} Q\right)
\end{aligned}
$$




$$
\begin{aligned}
& 0= 4 D_{\nu}\left(\mathcal{M}_{M P} \mathcal{M}_{N Q} \mathcal{H}^{(2) P Q \nu \mu}\right)-\frac{g}{2} X_{M N P} Q \mathcal{M}_{Q R} D^{\mu} \mathcal{M}^{P R} \\
&- 2 \epsilon_{M N P Q R} \mathcal{M}^{P S} \mathcal{H}^{(3) \mu \nu \rho}{ }_{S} \mathcal{H}_{\nu \rho}^{(2) Q R}+\frac{1}{9} e^{-1} \epsilon^{\mu \nu \rho \lambda \sigma \tau \kappa} \mathcal{H}_{\nu \rho \lambda M}^{(3)} \mathcal{H}_{\sigma \tau \kappa N}^{(3)}, \\
& 0= D_{\rho}\left(\mathcal{M}^{M N} \mathcal{H}^{(3) \rho \mu \nu}{ }_{N}\right)-2 g Z^{N P, M} \mathcal{M}_{N Q} \mathcal{M}_{P R} \mathcal{H}^{(2) Q R \mu \nu} \\
&-\frac{1}{3} e^{-1} \epsilon^{\mu \nu \rho \lambda \sigma \tau \kappa} \mathcal{H}_{\rho \lambda}^{(2) M N} \mathcal{H}_{\sigma \tau \kappa N}^{(3)}, \\
& 0=e^{-1} \epsilon^{\mu \nu \rho \lambda \sigma \tau \kappa} Y_{M N} \mathcal{H}_{\lambda \sigma \tau \kappa}^{(4) N}-6 Y_{M N} \mathcal{M}^{N P} \mathcal{H}^{(3) \mu \nu \rho}{ }_{P} .
\end{aligned}
$$

\section{APPENDIX B: TRUNCATION ANSATZE}

In this appendix, we collect relevant formulas for truncations of 11-dimensional supergravity on $S^{4}$ and type IIA theory on $S^{3}$. These give rise to $S O(5)$ and $C S O(4,0,1)$ gauged supergravities in seven dimensions, respectively. The complete $S^{4}$ truncation of 11-dimensional supergravity has been constructed in $[29,30]$ while the $S^{3}$ truncation of type IIA theory has been given in [31]. For both truncations, we will use the convention of [31].

\section{The 11-dimensional supergravity on $S^{4}$}

The ansatz for the 11-dimensional metric is given by

$$
d \hat{s}_{11}^{2}=\Delta^{\frac{1}{3}} d s_{7}^{2}+\frac{1}{\hat{g}^{2}} \Delta^{-\frac{2}{3}} T_{M N}^{-1} D \mu^{M} D \mu^{N}
$$

with the coordinates $\mu^{M}, M=1,2,3,4,5$, on $S^{4}$ satisfying $\mu^{M} \mu^{M}=1$. $T_{M N}$ is a unimodular $5 \times 5$ symmetric matrix describing scalar fields in the $S L(5) / S O(5)$ coset. The warped factor is defined by

$$
\Delta=T_{M N} \mu^{M} \mu^{N}
$$

The ansatz for the four-form field strength reads

$$
\begin{aligned}
\hat{F}_{(4)} & =\frac{1}{\hat{g}^{3}} \Delta^{-2}\left[\frac{1}{3 !} \epsilon_{M_{1} \ldots M_{5}} \mu^{M} \mu^{N} T^{M_{1} M} D T^{M_{2} N} \wedge D \mu^{M_{3}} \wedge D \mu^{M_{4}} \wedge D \mu^{M_{5}}\right] \\
& -\frac{1}{\hat{g}^{3}} \Delta^{-2} \mathcal{U} \epsilon_{(4)}+\frac{1}{4 \hat{g}^{2}} \Delta^{-1} \epsilon_{M_{1} \ldots M_{5}} F_{(2)}^{M_{1} M_{2}} \wedge D \mu^{M_{3}} \wedge D \mu^{M_{4}} T^{M_{5} N} \mu^{N} \\
& +\frac{1}{\hat{g}} \tilde{S}_{(3)}^{M} \wedge D \mu^{M}-T_{M N} * \tilde{S}_{(3)}^{M} \mu^{N} .
\end{aligned}
$$

In these equations, we have used the following definitions:

$$
\begin{gathered}
\mathcal{U}=2 T_{M N} T_{N P} \mu^{M} \mu^{P}-\Delta T_{M M}, \\
\epsilon_{(4)}=\frac{1}{4 !} \epsilon_{M_{1} \cdots M_{5}} \mu^{M_{1}} D \mu^{M_{2}} \wedge D \mu^{M_{3}} \wedge D \mu^{M_{4}} \wedge D \mu^{M_{5}},
\end{gathered}
$$

$$
D \mu^{M}=d \mu^{M}+\hat{g} \tilde{A}_{(1)}^{M N} \mu^{N}, \quad F_{(2)}^{M N}=d \tilde{A}_{(1)}^{M N}+\hat{g} \tilde{A}_{(1)}^{M P} \wedge \tilde{A}_{(1)}^{P N},
$$

$$
D T_{M N}=d T_{M N}+\hat{g} \tilde{A}_{(1)}^{M P} T_{P N}+\hat{g} \tilde{A}_{(1)}^{N P} T_{M P} .
$$

We have denoted the vector and massive three-form fields by $\tilde{A}_{(1)}^{M N}$ and $\tilde{S}_{(3)}^{M}$ to avoid confusion with those appearing in (22).

To find the identification between the seven-dimensional fields and parameters obtained from the $S^{4}$ truncation and those in seven-dimensional gauged supergravity of [23], we consider the kinetic terms of various fields and the scalar potential. After being multiplied by $\frac{1}{2}$, the relevant terms in the seven-dimensional Lagrangian of [31] can be written as 


$$
\begin{aligned}
e^{-1} \mathcal{L}_{S^{4}} & =\frac{1}{2} R+\frac{1}{8} D_{\mu} T_{M N}^{-1} D^{\mu} T_{M N}-\frac{1}{4} \hat{g}^{2}\left[2 T_{M N} T_{M N}-\left(T_{M M}\right)^{2}\right] \\
& -\frac{1}{16} T_{M P}^{-1} T_{N Q}^{-1} F_{\mu \nu}^{M N} F^{P Q \mu \nu}-\frac{1}{24} T_{M N} \tilde{S}_{\mu \nu \rho}^{M} \tilde{S}^{N \mu \nu \rho} .
\end{aligned}
$$

Comparing (22) with $Y_{M N}=\delta_{M N}, Z^{M N, P}=0$, we find the following identification:

$$
T_{M N}=\mathcal{M}^{M N}, \quad \tilde{S}_{(3)}^{M}=2 \mathcal{H}_{M}^{(3)}, \quad F_{(2)}^{M N}=4 \mathcal{H}_{(2)}^{M N}, \quad \hat{g}=\frac{1}{4} g
$$

\section{Type IIA supergravity on $S^{3}$}

The consistent truncation of type IIA supergravity on $S^{3}$ has been obtained in [31] by taking a degenerate limit of the $S^{4}$ truncation of 11-dimensional supergravity. To write down this truncation ansatz, we first split the index $M$ as $M=(i, 5), i=1,2,3,4$. The scalar matrix of $S L(5) / S O(5)$ coset is then given by

$$
T_{M N}^{-1}=\left(\begin{array}{cc}
\Phi^{-\frac{1}{4}} M_{i j}^{-1}+\Phi \chi_{i} \chi_{j} & \Phi \chi_{i} \\
\Phi \chi_{j} & \Phi
\end{array}\right)
$$

where $M_{i j}$ is a unimodular $4 \times 4$ symmetric matrix describing the $S L(4) / S O(4)$ coset.

The ten-dimensional metric, dilaton, and field strength tensors of various form fields are given by

$$
\begin{gathered}
d \hat{s}_{10}^{2}=\Phi^{\frac{3}{16}} \Delta^{\frac{1}{4}} d s_{7}^{2}+\frac{1}{\hat{g}^{2}} \Phi^{-\frac{5}{16}} \Delta^{-\frac{3}{4}} M_{i j}^{-1} D \mu^{i} D \mu^{j}, \\
e^{2 \hat{\varphi}}=\Delta^{-1} \Phi^{\frac{5}{4}} \\
\hat{F}_{(2)}=G_{(1)}^{i} \wedge D \mu^{i}+\hat{g} \mu^{i} G_{(2)}^{i}, \\
\hat{H}_{(3)}=\frac{1}{\hat{g}^{3}} \Delta^{-2}\left[-\mathcal{U} \epsilon_{(3)}+\frac{1}{2} \epsilon_{i_{1} i_{2} i_{3} i_{4}} M_{i_{1} j} \mu^{j} \mu^{k} D M_{i_{2} k} \wedge D \mu^{i_{3}} \wedge D \mu^{i_{4}}\right] \\
+\frac{1}{2 \hat{g}^{2}} \Delta^{-1} \epsilon_{i j k l} M_{i m} \mu^{m} F_{(2)}^{j k} \wedge D \mu^{l}+\frac{1}{\hat{g}} \tilde{S}_{(3)}, \\
\hat{F}_{(4)}=\frac{1}{\hat{g}^{3}} \Delta^{-1} M_{i j} \mu^{j} G_{(1)}^{i} \wedge \epsilon_{(3)}+\frac{1}{2 \hat{g}^{2}} \Delta^{-1} \epsilon_{i_{1} i_{2} i_{3} i_{4}} M_{i_{4} j} \mu^{j} G_{(2)}^{i_{1}} \wedge D \mu^{i_{2}} \wedge D \mu^{i_{3}} \\
+M_{i j} \Phi^{\frac{1}{4}} \mu^{j} * G_{(3)}^{i}+\frac{1}{\hat{g}} G_{(3)}^{i} \wedge D \mu^{i}
\end{gathered}
$$

with

$$
\begin{gathered}
\epsilon_{(3)}=\frac{1}{3 !} \epsilon_{i j k l} \mu^{i} D \mu^{j} \wedge D \mu^{k} \wedge D \mu^{l}, \quad D \mu^{i}=d \mu^{i}+\hat{g} \tilde{A}_{(1)}^{i j} \mu^{j}, \\
\mathcal{U}=2 M_{i j} M_{j k} \mu^{i} \mu^{k}-\Delta M_{i i}, \quad \Delta=M_{i j} \mu^{i} \mu^{j}, \\
G_{(1)}^{i}=D \chi_{i}+\hat{g} \tilde{A}_{(1)}^{i 5}, \quad G_{(2)}^{i}=D \tilde{A}_{(1)}^{5 i}+\chi_{j} F_{(2)}^{j i}, \\
G_{(3)}^{i}=\tilde{S}_{(3)}^{i}-\chi_{i} \tilde{S}_{(3)}, \quad F_{(2)}^{i j}=d \tilde{A}_{(1)}^{i j}+\hat{g} \tilde{A}_{(1)}^{i k} \wedge \tilde{A}_{(1)}^{k j}, \\
\tilde{S}_{(3)}=d B_{(2)}+\frac{1}{8} \epsilon_{i j k l}\left(F_{(2)}^{i j} \wedge \tilde{A}_{(1)}^{k l}-\frac{1}{3} \hat{g} \tilde{A}_{(1)}^{i j} \wedge \tilde{A}_{(1)}^{k m} \wedge \tilde{A}_{(1)}^{m l}\right) .
\end{gathered}
$$

By comparing the truncated Lagrangian and the seven-dimensional gauged Lagrangian given in (22) with $Y_{i j}=\delta_{i j}$ and $Y_{55}=0$, we find the following relations: 


$$
\begin{aligned}
\Phi & =e^{8 \phi_{0}}, \quad \chi_{i}=b_{i}, \quad M_{i j}^{-1}=\widetilde{\mathcal{M}}_{i j}, \\
\hat{g} & =\frac{1}{4} g, \quad \tilde{S}_{(3)}^{i}=2 \mathcal{H}_{i}^{(3)}, \quad F_{(2)}^{i j}=4 \mathcal{H}_{(2)}^{i j}, \quad \tilde{F}_{(2)}^{i}=4 \mathcal{H}_{(2)}^{i 5} .
\end{aligned}
$$

In this case, $\mu^{i}$ are coordinates on $S^{3}$ satisfying $\mu^{i} \mu^{i}=1$.

[1] J. M. Maldacena, The large $N$ limit of superconformal field theories and supergravity, Adv. Theor. Math. Phys. 2, 231 (1998).

[2] S. S. Gubser, I. R. Klebanov, and A. M. Polyakov, Gauge theory correlators from non-critical string theory, Phys. Lett. B 428, 105 (1998).

[3] E. Witten, Anti De Sitter Space and holography, Adv. Theor. Math. Phys. 2, 253 (1998).

[4] H. J. Boonstra, K. Skenderis, and P. K. Townsend, The domain-wall/QFT correspondence, J. High Energy Phys. 01 (1999) 003.

[5] T. Gherghetta and Y. Oz, Supergravity, Non-conformal field theories and brane-worlds, Phys. Rev. D 65, 046001 (2002).

[6] I. Kanitscheider, K. Skenderis, and M. Taylor, Precision holography for non-conformal branes, J. High Energy Phys. 09 (2008) 094.

[7] M. Henningson and K. Skenderis, The holographic Weyl anomaly, J. High Energy Phys. 07 (1998) 023.

[8] S. de Haro, S. N. Solodukhin, and K. Skenderis, Holographic reconstruction of spacetime and renormalization in the AdS/CFT correspondence, Commun. Math. Phys. 217, 595 (2001).

[9] M. Bianchi, D. Z. Freedman, and K. Skenderis, How to go with an RG flow, J. High Energy Phys. 08 (2001) 041.

[10] M. Bianchi, D. Z. Freedman, and K. Skenderis, Holographic renormalization, Nucl. Phys. B631, 159 (2002).

[11] I. Kanitscheider, K. Skenderis, and M. Taylor, Precision holography for non-conformal branes, J. High Energy Phys. 09 (2008) 094.

[12] G. Dibitetto and N. Petri, BPS objects in $D=7$ supergravity and their M-theory origin, J. High Energy Phys. 12 (2017) 041.

[13] P. Karndumri and P. Nuchino, Supersymmetric solutions from matter-coupled $7 D N=2$ gauged supergravity, Phys. Rev. D 98, 086012 (2018).

[14] G. Dibitetto and N. Petri, 6d surface defects from massive type IIA, J. High Energy Phys. 01 (2018) 039.

[15] G. Dibitetto and N. Petri, Surface defects in the D4-D8 brane system, J. High Energy Phys. 01 (2019) 193.

[16] G. Dibitetto and N. Petri, $\mathrm{AdS}_{2}$ solutions and their massive IIA origin, J. High Energy Phys. 05 (2019) 107.

[17] O. DeWolfe, D. Z. Freedman, and H. Ooguri, Holography and defect conformal field theories, Phys. Rev. D 66, 025009 (2002).
[18] C. Bachas, J. de Boer, R. Dijkgraaf, and H. Ooguri, Permeable conformal walls and holography, J. High Energy Phys. 06 (2002) 027.

[19] O. Aharony, O. DeWolfe, D. Z. Freedman, and A. Karch, Defect conformal field theory and locally localized gravity, J. High Energy Phys. 07 (2003) 030.

[20] A. B. Clark, D. Z. Freedman, A. Karch, and M. Schnabl, Dual of the Janus solution: An interface conformal field theory, Phys. Rev. D 71, 066003 (2005).

[21] A. Kapustin, Wilson-t Hooft operators in four-dimensional gauge theories and S-duality, Phys. Rev. D 74, 025005 (2006).

[22] A. Clark and A. Karch, Super Janus, J. High Energy Phys. 10 (2005) 094.

[23] H. Samtleben and M. Weidner, The maximal $D=7$ supergravities, Nucl. Phys. 725, 383 (2005).

[24] M. Pernici, K. Pilch, and P. van Nieuwenhuizen, Gauged maximally extended supergravity in seven-dimensions, Phys. Lett. 143B, 103 (1984).

[25] M. Pernici, K. Pilch, P. van Nieuwenhuizen, and N.P. Warner, Noncompact gaugings and critical points of maximal supergravity in seven-dimensions, Nucl. Phys. B249, 381 (1985).

[26] P. Karndumri and P. Nuchino, Supersymmetric domain walls in $7 D$ maximal gauged supergravity, Eur. Phys. J. C 79, 648 (2019).

[27] O. Hohm and H. Samtleben, Consistent Kaluza-Klein truncations via exceptional field theory, J. High Energy Phys. 01 (2015) 131.

[28] E. Malek and H. Samtleben, Dualising consistent IIA /IIB truncations, J. High Energy Phys. 12 (2015) 029.

[29] H. Nastase, D. Vaman, and P. van Nieuwenhuizen, Consistent nonlinear KK reduction of $11-d$ supergravity on $\operatorname{AdS}(7) \times S(4)$ and selfduality in odd dimensions, Phys. Lett. B 469, 96 (1999).

[30] H. Nastase, D. Vaman, and P. van Nieuwenhuizen, Consistency of the $\mathrm{AdS}_{7} \times S^{4}$ reduction and the origin of selfduality in odd dimensions, Nucl. Phys. B581, 179 (2000).

[31] M. Cvetic, H. Lu, C. N. Pope, A. Sadrzadeh, and T. A. Tran, $S^{3}$ and $S^{4}$ reductions of type IIA supergravity, Nucl. Phys. B590, 233 (2000).

[32] O. Lunin, 1/2-BPS states in M theory and defects in the dual CFTs, J. High Energy Phys. 10 (2007) 014.

[33] E. A. Bergshoeff, A. Kleinschmidt, and F. Riccioni, Supersymmetric domain walls, Phys. Rev. D 86, 085043 (2012). 